\title{
Flux Parameter Spaces in Type II Vacua
}

\author{
Ching-Ming Chen, ${ }^{1}$ Tianjun Li, ${ }^{1,2}$ and Dimitri V. Nanopoulos ${ }^{1,3,4}$ \\ ${ }^{1}$ George P. and Cynthia W. Mitchell Institute for Fundamental Physics, \\ Texas A\&M University, College Station, TX 77843, USA \\ ${ }^{2}$ Institute of Theoretical Physics, Chinese Academy of Sciences, Beijing 100080, China \\ ${ }^{3}$ Astroparticle Physics Group, Houston Advanced Research Center (HARC), \\ Mitchell Campus, Woodlands, TX r7381, USA \\ ${ }^{4}$ Academy of Athens, Division of Natural Sciences, \\ 28 Panepistimiou Avenue, Athens 10679, Greece
}

(Dated: November 6, 2018)

\begin{abstract}
We study the flux parameter spaces for semi-realistic supersymmetric Pati-Salam models in the AdS vacua on Type IIA orientifold and realistic supersymmetric Pati-Salam models in the Minkowski vacua on Type IIB orientifold. Because the fluxes can be very large, we show explicitly that there indeed exists a huge number of semi-realistic Type IIA and realistic Type IIB flux models. In the Type IIA flux models, in the very large flux limit, the theory can become weakly coupled and the AdS vacua can approach to the Minkowski vacua. In a series of realistic Type IIB flux models, at the string scale, the gauge symmetry can be broken down to the Standard Model (SM) gauge symmetry, the gauge coupling unification can be achieved naturally, all the extra chiral exotic particles can be decoupled, and the observed SM fermion masses and mixings can be obtained as well. In particular, the real parts of the dilaton, Kähler moduli, and the unified gauge coupling are independent of the very large fluxes. The very large fluxes only affect the real and/or imaginary parts of the complex structure moduli, and/or the imaginary parts of the dilaton and Kähler moduli. However, these semi-realistic Type IIA and realistic Type IIB flux models can not be populated in the string landscape.
\end{abstract}

PACS numbers: 11.10.Kk, 11.25.Mj, 11.25.-w, 12.60.Jv 


\section{INTRODUCTION}

One of the most challenging problems in string theory is the construction of realistic string vacua, which can give us the low-energy supersymmetric Standard Model (SM) and stabilize the moduli fields. Such constructions will give us a bridge between string theory and low-energy realistic particle physics. With M-theory, we can probe the physical string vacua not only in perturbative heterotic string theory, but also in perturbative Type I, Type IIA and Type IIB superstring theory. Especially, because of the advent of D-branes, we can construct consistent four-dimensional $N=1$ supersymmetric chiral models with non-Abelian gauge symmetry on Type II orientifolds, by employing conformal field theory techniques in the open string sector [1].

During the last few years, Type II orientifolds with intersecting D-branes have been highly interesting in the string model building where the chiral fermions come from the intersections of the D-branes in the internal space [2] with T-dual decription in terms of magnetized D-branes [3]. On Type IIA orientifolds with intersecting D6-branes, a large number of non-supersymmetric three-family Standard-like models and Grand Unified Theories (GUTs), which satisfy the Ramond-Ramond (RR) tadpole cancellation conditions, were constructed [4, 5, 6, 7]. However, there generically exist the uncancelled Neveu-SchwarzNeveu-Schwarz (NSNS) tadpoles and the gauge hierarchy problem. To solve these problems, the semi-realistic supersymmetric standard-like models and GUTs have been constructed in Type IIA theory on $T^{6} /\left(\mathbf{Z}_{\mathbf{2}} \times \mathbf{Z}_{\mathbf{2}}\right)$ orientifold [8, 9, 10, 11, 12, 13, 14, 15] and other orientifolds [16]. In particular, only the Pati-Salam models can give us all the SM fermion Yukawa couplings at the stringy tree level, and we can explain the SM fermion masses and mixings in one model [17, 18].

Although some of the complex structure moduli (in Type IIA picture) and the dilaton field might be stabilized due to the gaugino condensation in the hidden sector in some models (for example, see Ref. [19]), the moduli stabilization is still a big challenge. Recently, important progresses have been made by introducing background fluxes. In Type IIB theory, the RR fluxes and NSNS fluxes generate a superpotential [20] that depends on the dilaton and complex structure moduli, and then stabilize these fields dynamically [21, 22]. With non-perturbative effects, one can further determine the Kähler moduli [23]. For such kind of model building, the RR and NSNS fluxes contribute to large positive D3-brane charges due to the Dirac quantization [24, 25]. Thus, they modify the global RR tadpole cancellation conditions significantly and impose strong constraints on the consistent model building [26, 27, 28, 29, 30]. In addition, turning on the RR, NSNS, metric, and non-geometric fluxes [31, 32, 33], we can stabilize close string moduli in supersymmetric Minkowski vacua [34]. In particular, these fluxes can contribute negative D-brane charges 
to the RR tadpole cancellation conditions, and then we can relax the RR tadpole cancellation conditions elegantly [34]. In Type IIA theory with RR, NSNS, and metric fluxes [35, 36, 37], we can stabilize the moduli in supersymmetric AdS vacua as well as relax the RR tadpole cancellation conditions [37, 38]. Interestingly, by relaxing the RR tadpole cancellation conditions, we can construct semi-realistic Type IIA [38, 39] and realistic IIB [34] Pati-Salam flux models that can explain the SM fermion masses and mixings.

One of the most interesting consequences from Type II flux compactifications is that there may exist a large number of meta-stable vacua [40, 41]. The ensemble of these vacua is called string landscape [40]. With the "weak anthropic principle" [42], this proposal may provide the first concrete explanation of the very tiny value of the cosmological constant which can take only the discrete values. Because the fluxes can contribute negative D-brane charges to the RR tadpole cancellation conditions in Type IIA and IIB theories with general flux compactifications [34, 37, 38], we can easily construct many (or even infinite) Type IIA and IIB flux vacua by increasing the corresponding fluxes. Therefore, the remain interesting open questions are whether we can have a huge number of semi-realistic Type IIA and realistic Type IIB flux vacua, and whether these vacua can be populated in the string landscape.

In this paper, we shall study the flux parameter spaces in details for semi-realistic supersymmetric Pati-Salam models in the AdS vacua on Type IIA orientifold and realistic supersymmetric Pati-Salam models in the Minkowski vacua on Type IIB orientifold with general flux compactifications. We show explicitly that there indeed exists a huge number of semi-realistic Type IIA and realistic Type IIB Pati-Salam flux models. These discussions also confirm the possibility of the string landscape. However, it seems to us that these semirealistic Type IIA and realistic Type IIB flux models can not be populated in the string landscape. The point is the following: we can easily construct much more models that do not have the SM gauge symmetry while satisfy the same model building constraints, for concrete examples, see the statistical study in Ref. [43].

First, we briefly review the supersymmetric intersecting D6-brane model building in the AdS vacua on Type IIA orientifold with flux compactifications. In general, even though we take the RR fluxes $e$ and/or $e_{0}$ (for detail definitions, see the following discussions and those in Ref. [37]) to be very large, we do not change the gauge groups and the particle spectra of the D6-brane models since the fluxes $e$ and $e_{0}$ do not contribute to the RR tadpole cancellation conditions. In particular, if we take both $e$ and $e_{0}$ to be very large while keeping $\left(e_{0} a-e h_{0}\right)$ as a constant where $a$ and $h_{0}$ are respectively the metric and NSNS fluxes, the very large fluxes $e$ and $e_{0}$ will not affect the dilaton $s$, Kähler moduli $t_{i}$, complex structure moduli $u_{i}$, and the cosmological constant of the models. In this case, we can take $e$ and $e_{0}$ to be very large, and keep the same main properties of the models, for example, the gauge symmetries, the gauge coupling constants, the particle spectra, and the 
cosmological constant, etc. The only difference is that one linear combination of imagininary parts of $s$ and $u_{i}\left(3 a \operatorname{Im} s+\sum_{i=1}^{3} b_{i} \operatorname{Im} u_{i}\right)$ will be very large and proportional to $e$ where $b_{i}$ are metric fluxes. Moreover, if $\left(e_{0} a-e h_{0}\right)$ is very large, we show that the theory will be very weakly coupled, and the magnitude of the cosmological constant will be very small and proportional to $\left(e_{0} a-e h_{0}\right)^{-5 / 3}$ so that the AdS vacua approach to the Minkowski vacua. And if only $e_{0}$ is very large, $\left(3 a \operatorname{Im} s+\sum_{i=1}^{3} b_{i} \operatorname{Im} u_{i}\right)$ will be a constant as well since it only depends on $e$. Next, we take the other fluxes $a, h_{0}, m$, and/or $q$ to be very large where $m$ and $q$ are RR fluxes. Interestingly, only the quadratic combination $\left(h_{0} m+3 q a\right)$ appears in the RR tadpole cancellation conditions. And if $\left(h_{0} m+3 q a\right)<0$, the fluxes contribute negative D-brane charges to all the RR tadpole cancellation conditions. Thus, when $a, h_{0}$, $m$, and/or $q$ are very large, we consider two kinds of models: (1) $\left(h_{0} m+3 q a\right)$ is a constant; (2) $\left(h_{0} m+3 q a\right)$ is very large. In the first kind of Pati-Salam models which was constructed and studied by us previously [38], we take $h_{0}, m$, and $q$ to be very large while keeping $\left(h_{0} m+3 q a\right)$ as a constant. Thus, we do not change the RR tadpole concellation conditions, the gauge symmetries, and the particle spectra of the models. In the very large flux limit, the theory becomes very weakly coupled, the magnitude of the cosmological constant will become very small, and then the AdS vacua also approach to the Minkowski vacua. In the second kind of Pati-Salam models, we not only take $a$ and $h_{0}$ to be very large, but also take $\left(h_{0} m+3 q a\right)$ to be very large. If $\left(h_{0} m+3 q a\right)$ is negative and very large, we can always satisfy the RR tadpole cancellation conditions by introducing more D-branes. However, we will have many chiral exotic particles that can not be decoupled easily. Thus, we take the complex structure moduli to be very large so that only one of the RR tadpole cancellation conditions will become very large and proportional to $a$ or $h_{0}$. We show that at the string scale, the gauge symmetry can be broken down to the SM gauge symmetry, and the exotic particles might be decoupled. The gauge couplings for $S U(2)_{L}$ and $S U(2)_{R}$ are half of these for $S U(3)_{C}$ and $U(1)_{B-L}$. In the very large flux limit, the gauge coulings approach to the fixed constants, but the magnitude of the cosmological constant will be very large which can be made very small again if we introduce very large $e$ and/or $e_{0}$ fluxes.

Second, we briefly review the supersymmetric D-brane model building in the Minkowski vacua on Type IIB orientifold with general flux compactifications. We construct a series of realistic Pati-Salam models with gauge groups $U(4)_{C} \times U(2)_{L} \times U(2)_{R}$ and $U S p(10) \times$ $U S p(6(\kappa-1))^{3}$ in the observable sector and hidden sector, respectively, where $\kappa=1,2,3, \ldots$. At the string scale, the gauge symmetry can be broken down to the SM gauge symmetry, the gauge coupling unification can be achieved naturally, and all the extra chiral exotic particles can be decoupled so that we have the supersymmetric SMs with/without SM singlet(s) below the string scale. The observed SM fermion masses and mixings can also be obtained. In addition, the unified gauge coupling, the dilaton, the complex structure moduli, the real 
parts of the Kähler moduli and the sum of the imaginary parts of the Kähler moduli can be determined as functions of the four-dimensional dilaton and fluxes, and can be estimated as well. In particular, in the very large flux limit, we can choose the unified gauge coupling at the string scale as the unified gauge coupling in the supersymmetric SMs at the GUT scale. In other words, we can choose the real parts of the dilaton $(s)$ and Kähler moduli $\left(t_{i}\right)$ as constants because they are independent of the fluxes. And we emphasize that the cosmological constant is always zero in these models due to the Minkowski vacua. Moreover, we consider the flux parameter spaces in two kinds of the models: $\kappa=1$ and $\kappa>1$. For the first kind of models with $\kappa=1$, we do not have gauge symmetries $U S p(6(\kappa-1))^{3}$, and we have constructed and studied such kind of models previously [34]. In these models, the very large fluxes do not contribute to the RR tadpole cancellation conditions, and we find two kinds of solutions to the flux consistent equations. In the very large flux limit, the real part of the complex structure moduli $\left(u_{1}=u_{2}=u_{3} \equiv u\right)$ and the sum of the imaginary parts of the Kähler moduli $\left(t \equiv t_{1}+t_{2}+t_{3}\right)$ are constants and independent of the very large fluxes. Only the imaginary parts of the dilaton and complex structure moduli will be very large and proportional to the very large fluxes. For the second kind of models with $\kappa>1$, we obtain four kinds of solutions to the flux consistent equations, and show that there indeed exist a huge number of the realistic flux vacua. In the very large $\kappa$ limit, we consider the asymptotic behaviour for $\operatorname{Re} u, \operatorname{Im} s, \operatorname{Im} t$, and $\operatorname{Im} u$, and present some concrete examples.

This paper is organized as follows. In Section II, we briefly review the supersymmetric intersecting D6-brane model building in the AdS vacua on Type IIA orientifold with flux compactifications and the general case with very large fluxes $e$ and/or $e_{0}$. And we study two kinds of the Type IIA Pati-Salam flux models in Section III. In Section IV, we briefly review the supersymmetric D-brane model building in the Minkowski vacua on Type IIB orientifold with general flux compactifications. We study a series of realistic Pati-Salam flux models in Section V. Discussion and conclusions are given in Section VI.

\section{FLUX MODEL BUILDING ON TYPE IIA ORIENTIFOLD}

Let us briefly review the rules for the intersecting D6-brane model building in Type IIA theory on $\mathbf{T}^{\mathbf{6}}$ orientifold with flux compactifications [36, 37]. We consider $\mathbf{T}^{\mathbf{6}}$ to be a sixtorus factorized as $\mathbf{T}^{\mathbf{6}}=\mathbf{T}^{2} \times \mathbf{T}^{2} \times \mathbf{T}^{2}$ whose complex coordinates are $z_{i}, i=1,2,3$ for the $i$-th two-torus, respectively. We implement an orientifold projection $\Omega R$, where $\Omega$ is the world-sheet parity, and $R$ acts on the complex coordinates as

$$
R:\left(z_{1}, z_{2}, z_{3}\right) \rightarrow\left(\bar{z}_{1}, \bar{z}_{2}, \bar{z}_{3}\right) .
$$


Thus, we have the orientifold 6-planes (O6-planes) under the actions of $\Omega R$. In addition, we introduce some stacks of D6-branes which wrap on the factorized three-cycles. There are two kinds of complex structures consistent with orientifold projection for a two-torus rectangular and tilted [9, 44]. If we denote the homology classes of the three cycles wrapped by $a$ stack of $N_{a}$ D6-branes as $n_{a}^{i}\left[a_{i}\right]+m_{a}^{i}\left[b_{i}\right]$ and $n_{a}^{i}\left[a_{i}^{\prime}\right]+\tilde{m}_{a}^{i}\left[b_{i}\right]$ with $\left[a_{i}^{\prime}\right]=\left[a_{i}\right]+\frac{1}{2}\left[b_{i}\right]$ for the rectangular and tilted two-tori respectively, we can label a generic one cycle by $\left(n_{a}^{i}, l_{a}^{i}\right)$ in which $l_{a}^{i} \equiv m_{a}^{i}$ for a rectangular two-torus while $l_{a}^{i} \equiv 2 m_{a}^{i}=2 \tilde{m}_{a}^{i}+n_{a}^{i}$ for a tilted twotorus [11]. For $a$ stack of $N_{a}$ D6-branes along the cycle $\left(n_{a}^{i}, l_{a}^{i}\right)$, we also need to include their $\Omega R$ images $N_{a^{\prime}}$ with wrapping numbers $\left(n_{a}^{i},-l_{a}^{i}\right)$. For the D6-branes on the top of O6planes, we count them and their $\Omega R$ images independently. So, the homology three-cycles for $a$ stack of $N_{a}$ D6-branes and its orientifold image $a^{\prime}$ are

$$
\left[\Pi_{a}\right]=\prod_{i=1}^{3}\left(n_{a}^{i}\left[a_{i}\right]+2^{-\beta_{i}} l_{a}^{i}\left[b_{i}\right]\right), \quad\left[\Pi_{a^{\prime}}\right]=\prod_{i=1}^{3}\left(n_{a}^{i}\left[a_{i}\right]-2^{-\beta_{i}} l_{a}^{i}\left[b_{i}\right]\right),
$$

where $\beta_{i}=0$ if the $i$-th two-torus is rectangular and $\beta_{i}=1$ if it is tilted. And the homology three-cycle wrapped by the O6-planes is

$$
\Omega R:\left[\Pi_{O 6}\right]=2^{3}\left[a_{1}\right] \times\left[a_{2}\right] \times\left[a_{3}\right] .
$$

Therefore, the intersection numbers are

$$
\begin{gathered}
I_{a b}=\left[\Pi_{a}\right]\left[\Pi_{b}\right]=2^{-k} \prod_{i=1}^{3}\left(n_{a}^{i} l_{b}^{i}-n_{b}^{i} l_{a}^{i}\right), \\
I_{a b^{\prime}}=\left[\Pi_{a}\right]\left[\Pi_{b^{\prime}}\right]=-2^{-k} \prod_{i=1}^{3}\left(n_{a}^{i} l_{b}^{i}+n_{b}^{i} l_{a}^{i}\right), \\
I_{a a^{\prime}}=\left[\Pi_{a}\right]\left[\Pi_{a^{\prime}}\right]=-2^{3-k} \prod_{i=1}^{3}\left(n_{a}^{i} l_{a}^{i}\right), \\
I_{a O 6}=\left[\Pi_{a}\right]\left[\Pi_{O 6}\right]=-2^{3-k} l_{a}^{1} l_{a}^{2} l_{a}^{3},
\end{gathered}
$$

where $k=\beta_{1}+\beta_{2}+\beta_{3}$ is the total number of tilted two-tori.

For $a$ stack of $N_{a}$ D6-branes whose homology three-cycles are not invariant under $\Omega R$, we have $U\left(N_{a}\right)$ gauge symmetry. Otherwise, we obtain $U S p\left(2 N_{a}\right)$ gauge symmetry. The general spectrum of D6-branes' intersecting at generic angles, which is valid for both rectangular and tilted two-tori, is given in Table I. The four-dimensional $N=1$ supersymmetric models on Type IIA orientifolds with intersecting D6-branes are mainly constrained in two aspects: four-dimensional $N=1$ supersymmetry conditions, and RR tadpole cancellation conditions. 


\begin{tabular}{|c||c|}
\hline Sector & Representation \\
\hline \hline$a a$ & $U\left(N_{a}\right)$ vector multiplet and 3 adjoint chiral multiplets \\
\hline$a b+b a$ & $I_{a b}\left(N_{a}, \overline{N_{b}}\right)$ chiral multiplets \\
\hline$a b^{\prime}+b^{\prime} a$ & $I_{a b^{\prime}}\left(N_{a}, N_{b}\right)$ chiral multiplets \\
\hline$a a^{\prime}+a^{\prime} a$ & $\frac{1}{2}\left(I_{a a^{\prime}}+I_{a O 6}\right)$ anti-symmetric chiral multiplets \\
& $\frac{1}{2}\left(I_{a a^{\prime}}-I_{a O 6}\right)$ symmetric chiral multiplets \\
\hline
\end{tabular}

TABLE I: The general spectrum for the intersecting D6-brane model building in Type IIA theory on $\mathbf{T}^{\mathbf{6}}$ orientifold with flux compactifications.

To simplify the notation, we define the following products of wrapping numbers

$$
\begin{gathered}
A_{a} \equiv-n_{a}^{1} n_{a}^{2} n_{a}^{3}, \quad B_{a} \equiv n_{a}^{1} l_{a}^{2} l_{a}^{3}, \quad C_{a} \equiv l_{a}^{1} n_{a}^{2} l_{a}^{3}, \quad D_{a} \equiv l_{a}^{1} l_{a}^{2} n_{a}^{3} \\
\tilde{A}_{a} \equiv-l_{a}^{1} l_{a}^{2} l_{a}^{3}, \tilde{B}_{a} \equiv l_{a}^{1} n_{a}^{2} n_{a}^{3}, \tilde{C}_{a} \equiv n_{a}^{1} l_{a}^{2} n_{a}^{3}, \tilde{D}_{a} \equiv n_{a}^{1} n_{a}^{2} l_{a}^{3} .
\end{gathered}
$$

(1) Four-Dimensional $N=1$ Supersymmetry Conditions

The four-dimensional $N=1$ supersymmetry can be preserved by the orientation projection $(\Omega R)$ if and only if the rotation angle of any D6-brane with respect to any O6-plane is an element of $S U(3)$ [2], i. e., $\theta_{1}+\theta_{2}+\theta_{3}=0 \bmod 2 \pi$, where $\theta_{i}$ is the angle between the $D 6$-brane and the O6-plane on the $i$-th two-torus. Then the supersymmetry conditions can be rewritten as [11]

$$
\begin{gathered}
x_{A} \tilde{A}_{a}+x_{B} \tilde{B}_{a}+x_{C} \tilde{C}_{a}+x_{D} \tilde{D}_{a}=0, \\
A_{a} / x_{A}+B_{a} / x_{B}+C_{a} / x_{C}+D_{a} / x_{D}<0,
\end{gathered}
$$

where $x_{A}=\lambda, x_{B}=\lambda 2^{\beta_{2}+\beta 3} / \chi_{2} \chi_{3}, x_{C}=\lambda 2^{\beta_{1}+\beta 3} / \chi_{1} \chi_{3}$, and $x_{D}=\lambda 2^{\beta_{1}+\beta 2} / \chi_{1} \chi_{2}$ in which $\chi_{i}=R_{i}^{2} / R_{i}^{1}$ are the complex structure parameters and $\lambda$ is a positive real number.

(2) $R R$ Tadpole Cancellation Conditions

The total RR charges from the D6-branes and O6-planes and from the metric, NSNS, and RR fluxes must vanish since the RR field flux lines are conserved. With the filler branes on the top of the O6-plane, we obtain the RR tadpole cancellation conditions [36, 37]:

$$
\begin{gathered}
2^{k} N_{O 6}-\sum_{a} N_{a} A_{a}+\frac{1}{2}\left(m h_{0}+q_{1} a_{1}+q_{2} a_{2}+q_{3} a_{3}\right)=16 \\
\sum_{a} 2^{-\beta_{2}-\beta 3} N_{a} B_{a}+\frac{1}{2}\left(m h_{1}-q_{1} b_{11}-q_{2} b_{21}-q_{3} b_{31}\right)=0
\end{gathered}
$$




$$
\begin{aligned}
& \sum_{a} 2^{-\beta_{1}-\beta_{3}} N_{a} C_{a}+\frac{1}{2}\left(m h_{2}-q_{1} b_{12}-q_{2} b_{22}-q_{3} b_{32}\right)=0 \\
& \sum_{a} 2^{-\beta_{1}-\beta_{2}} N_{a} D_{a}+\frac{1}{2}\left(m h_{3}-q_{1} b_{13}-q_{2} b_{23}-q_{3} b_{33}\right)=0
\end{aligned}
$$

where $2 N_{O 6}$ are the number of filler branes wrapping along the O6-plane. In addition, $a_{i}$ and $b_{i j}$ arise from the metric fluxes, $h_{0}$ and $h_{i}$ arise from the NSNS fluxes, and $m$ and $q_{i}$ arise from the RR fluxes. We consider these fluxes $\left(a_{i}, b_{i j}, h_{0}, h_{i}, m\right.$ and $\left.q_{i}\right)$ quantized in units of 2 so that we can avoid the subtle problems with flux Dirac quantization conditions.

In addition to the above $\mathrm{RR}$ tadpole cancellation conditions, the discrete D-brane RR charges classified by $\mathbf{Z}_{2}$ K-theory groups in the presence of orientifolds, which are subtle and invisible by the ordinary homology [26, 45, 46], should also be taken into account [24]. The K-theory conditions for a $\mathbf{Z}_{\mathbf{2}} \times \mathbf{Z}_{\mathbf{2}}$ orientifold are

$$
\sum_{a} \tilde{A}_{a}=\sum_{a} \tilde{B}_{a}=\sum_{a} \tilde{C}_{a}=\sum_{a} \tilde{D}_{a}=0 \bmod 2
$$

Moreover, the Freed-Witten anomaly [47] cancellation condition is [37]

$$
-2^{-k} h_{0} \tilde{A}_{a}+2^{-\beta_{1}} h_{1} \tilde{B}_{a}+2^{-\beta_{2}} h_{2} \tilde{C}_{a}+2^{-\beta_{3}} h_{3} \tilde{D}_{a}=0
$$

Furthermore, there are seven moduli fields in the supergravity theory basis, the dilaton $s$, three Kähler moduli $t_{i}$, and three complex structure moduli $u_{i}$. And their real parts are

$$
\operatorname{Re} s \equiv \frac{e^{-\phi_{4}}}{\sqrt{\chi_{1} \chi_{2} \chi_{3}}}, \operatorname{Ret}_{i} \equiv \frac{A_{i}}{\alpha^{\prime}}, \operatorname{Re} u_{i} \equiv e^{-\phi_{4}} \sqrt{\frac{\chi_{j} \chi_{k}}{\chi_{i}}}
$$

where $\phi_{4}$ is the four-dimensional T-duality invariant dilaton, $A_{i}$ is the area for the $i$-th twotorus, $\alpha^{\prime}$ is string tension, and $i \neq j \neq k \neq i$. Moreover, $\phi_{4}$ is related to the ten-dimensional dilaton $\phi$ as following

$$
e^{\phi_{4}}=\frac{e^{\phi}}{\sqrt{\operatorname{Ret}_{1} \operatorname{Ret}_{2} \operatorname{Ret}_{3}}} .
$$

And the four-dimensional Planck scale $M_{\mathrm{Pl}}$ is

$$
M_{\mathrm{Pl}}=\frac{e^{-2 \phi_{4}}}{\pi \alpha^{\prime}} .
$$

Moreover, the full superpotential is [37]

$$
\begin{aligned}
\mathcal{W}= & e_{0}+i h_{0} S+\sum_{i=1}^{3}\left[\left(i e_{i}-a_{i} s-b_{i i} u_{i}-\sum_{j \neq i} b_{i j} u_{j}\right) t_{i}-i h_{i} u_{i}\right] \\
& -q_{1} t_{2} t_{3}-q_{2} t_{1} t_{3}-q_{3} t_{1} t_{2}+i m t_{1} t_{2} t_{3}
\end{aligned}
$$


where $e_{0}$ and $e_{i}$ are RR fluxes. The Kähler potential for these moduli is

$$
\mathcal{K}=-\ln (s+\bar{s})-\sum_{i=1}^{3} \ln \left(t_{i}+\bar{t}_{i}\right)-\sum_{i=1}^{3} \ln \left(u_{i}+\bar{u}_{i}\right) .
$$

And the holomorphic gauge kinetic function for a generic stack of D6-branes is given by [48, 49]

$$
\begin{aligned}
f_{a}= & \frac{1}{\kappa_{a}}\left(n_{a}^{1} n_{a}^{2} n_{a}^{3} s-n_{a}^{1} m_{a}^{2} m_{a}^{3} u_{1}\right. \\
& \left.-n_{a}^{2} m_{a}^{1} m_{a}^{3} u_{2}-n_{a}^{3} m_{a}^{1} m_{a}^{2} u_{3}\right)
\end{aligned}
$$

where $\kappa_{a}$ is equal to 1 and 2 for $U(n)$ and $U S p(2 n)$, respectively. We emphasize that $m_{a}^{i}$ is equal to $l_{a}^{i}$ and $l_{a}^{i} / 2$ for the rectangular and tilted two-torus, respectively.

In addition, the supergravity scalar potential is

$$
V=e^{\mathcal{K}}\left(\mathcal{K}^{i \bar{j}} D_{i} \mathcal{W} D_{\bar{j}} \mathcal{W}-3|\mathcal{W}|^{2}\right)
$$

where $\mathcal{K}^{i \bar{j}}$ is the inverse metric of $\mathcal{K}_{i \bar{j}} \equiv \partial_{i} \partial_{\bar{j}} \mathcal{K}, D_{i} \mathcal{W}=\partial_{i} \mathcal{W}+\left(\partial_{i} \mathcal{K}\right) \mathcal{W}$, and $\partial_{i}=\partial_{\phi_{i}}$ where $\phi_{i}$ can be $s, t_{i}$, and $u_{i}$.

In this paper, we concentrate on the supersymmetric AdS vacua [37]. For simplicity, we assume that the Kähler moduli $t_{i}$ satisfy $t_{1}=t_{2}=t_{3}$, then we obtain $q_{1}=q_{2}=q_{3} \equiv q$ and $e_{1}=e_{2}=e_{3} \equiv e$ at the AdS vacua. To satisfy the Jacobi identities for metric fluxes, we consider the solution $a_{i}=a, b_{i i}=-b_{i}$, and $b_{j i}=b_{i}$ in which $j \neq i$ [37]. Then, the superpotential becomes

$$
\mathcal{W}=e_{0}+3 i e t-3 q t^{2}+i m t^{3}+i h_{0} s-3 a s t-\sum_{k=0}^{3}\left(i h_{k}+b_{k} t\right) u_{k} .
$$

For the supersymmetric AdS vacua, we have

$$
D_{s} \mathcal{W}=D_{t} \mathcal{W}=D_{u_{i}} \mathcal{W}=0
$$

From the above equations, we obtain the following ten real equations for $m \neq 0[37]$

$$
\begin{gathered}
3 a \operatorname{Re} s=b_{i} \operatorname{Re} u_{i}, \quad \text { for } i=1,2,3, \\
3 h_{i} a+h_{0} b_{i}=0, \quad \text { for } i=1,2,3, \\
3 a \operatorname{Im} s+\sum_{i=1}^{3} b_{i} \operatorname{Im} u_{i}=3 e-\frac{3 q}{a}\left(3 h_{0}-7 a \operatorname{Im} t\right)-\frac{3 m}{a} \operatorname{Im} t\left(3 h_{0}-8 a \operatorname{Im} t\right), \\
a \operatorname{Re} s=-2 \operatorname{Ret}(q+m \operatorname{Im} t),
\end{gathered}
$$




$$
\begin{gathered}
160 \lambda^{3}+186\left(\lambda_{0}-1\right) \lambda^{2}+27\left(\lambda_{0}-1\right)^{2} \lambda+\lambda_{0}^{2}\left(\lambda_{0}-3\right)+\frac{27 a^{2}}{m h_{0}^{3}}\left(e_{0} a-e h_{0}\right)=0, \\
3 a^{2}(\operatorname{Re} t)^{2}=5 h_{0}^{2} \lambda\left(\lambda+\lambda_{0}-1\right),
\end{gathered}
$$

where

$$
\operatorname{Im} t=\left(\lambda+\lambda_{0}\right) \frac{h_{0}}{3 a} \quad, \quad \lambda_{0}=-\frac{3 q a}{m h_{0}}
$$

And we require $\lambda\left(\lambda+\lambda_{0}-1\right)>0$. Also, Eq. (29) can be rewritten as

$$
3 a^{2} \operatorname{Re} s=-2 h_{0} m \lambda \operatorname{Re} t
$$

Interestingly, it can be shown that if Eqs. (9), (26), and (27) are satisfied, the Freed-Witten anomaly is automatically cancelled. Moreover, we obtain the cosmological constant

$$
V_{0}=-\frac{a b_{1} b_{2} b_{3} \lambda_{0}^{2}\left(16 \lambda+\lambda_{0}-1\right)}{1920 q^{2} \operatorname{Ret}^{3} \lambda^{3}} .
$$

From Eqs. (26) and (27), we obtain

$$
\begin{gathered}
b_{1}=\frac{3 a}{\chi_{2} \chi_{3}}, b_{2}=\frac{3 a}{\chi_{1} \chi_{3}}, b_{3}=\frac{3 a}{\chi_{1} \chi_{2}}, \\
h_{1}=-\frac{h_{0}}{\chi_{2} \chi_{3}}, h_{2}=-\frac{h_{0}}{\chi_{1} \chi_{3}}, h_{3}=-\frac{h_{0}}{\chi_{1} \chi_{2}} .
\end{gathered}
$$

Thus, the RR tadpole cancellation conditions can be rewritten as following

$$
\begin{aligned}
& 2^{k} N_{\text {O6 }}-\sum_{a} N_{a} A_{a}+\frac{1}{2}\left(h_{0} m+3 a q\right)=16, \\
& \sum_{a} 2^{-\beta_{2}-\beta 3} N_{a} B_{a}-\frac{1}{2 \chi_{2} \chi_{3}}\left(h_{0} m+3 a q\right)=0, \\
& \sum_{a} 2^{-\beta_{1}-\beta_{3}} N_{a} C_{a}-\frac{1}{2 \chi_{1} \chi_{3}}\left(h_{0} m+3 a q\right)=0, \\
& \sum_{a} 2^{-\beta_{1}-\beta_{2}} N_{a} D_{a}-\frac{1}{2 \chi_{1} \chi_{2}}\left(h_{0} m+3 a q\right)=0 .
\end{aligned}
$$

Therefore, only the flux quadratic combination $\left(h_{0} m+3 a q\right)$ appears in the RR tadpole cancellation condition. And if $\left(h_{0} m+3 a q\right)<0$, the supergravity fluxes contribute negative D6-brane charges to all the RR tadpole cancellation conditions, and then, the RR tadpole 
cancellation conditions give no constraints on the consistent model building because we can always introduce suitable supergravity fluxes and some stacks of D6-branes in the hidden sector to cancel the RR tadpoles.

In general, we can take the fluxes $e_{0}$ and $e$ as arbitrary large integers that are multiples of 2 , i.e., $e_{0}$ and $e$ can be very large. Here, for simplicity, we assume that the fluxes $h_{0}$, $m, q$ and $a$ are the finite fixed integers that satisfy the RR tadpole cancellation conditions. Interestingly, if both $e$ and $e_{0}$ are very large while $\left(e_{0} a-e h_{0}\right)$ is a constant, the very large fluxes $e$ and $e_{0}$ will not affect the dilaton, Kähler moduli, complex structure moduli, and the cosmological constant of the models. So, we can take $e$ and $e_{0}$ to be very large, and keep the same main properties of the models, for example, the gauge symmetries, the gauge coupling constants, the particle spectra, and the cosmological constant, etc. The only difference is that one linear combination of the imagininary parts of $s$ and $u_{i}\left(3 a \operatorname{Im} s+\sum_{i=1}^{3} b_{i} \operatorname{Im} u_{i}\right)$ will be very large and proportional to $e$. For the case with very large $\left(e_{0} a-e h_{0}\right)$, we define

$$
\Delta \equiv\left[-\frac{27 a^{2}}{160 m h_{0}^{3}}\left(e_{0} a-e h_{0}\right)\right]^{1 / 3} .
$$

If $e_{0}$ and/or $e$ are/is very large, $\Delta$ can be very large. In particular, if we fix $e$ and only allow $e_{0}$ to be very large, the value of $3 a \operatorname{Im} s+\sum_{i=1}^{3} b_{i} \operatorname{Im} u_{i}$ is fixed and will not be very large. In the large $\Delta$ limit, we obtain

$$
\operatorname{Re} t \simeq \frac{\sqrt{15}}{3}\left|\frac{h_{0} \Delta}{a}\right|, \quad \operatorname{Re} s \simeq-\frac{2 h_{0} m \Delta}{3 a^{2}} \operatorname{Re} t, \quad \operatorname{Re} u_{i} \simeq-\frac{2 h_{0} m \Delta}{a b_{i}} \operatorname{Re} t .
$$

Thus we have

$$
e^{\phi_{4}} \sim \Delta^{-2}, \quad e^{\phi} \sim \Delta^{-1 / 2}, g_{a, b, c}^{-2} \sim \Delta^{2} .
$$

And then, the theory will be very weakly coupled. The cosmological constant is

$$
V_{0} \simeq-\frac{a b_{1} b_{2} b_{3} \lambda_{0}^{2}}{120 q^{2} \operatorname{Re}^{3} \lambda^{2}} \sim \Delta^{-5}
$$

So, the magnitude of the cosmological constant will be very small if $\Delta$ is very large. And then the AdS vacua will approach to the Minkowski vacua.

\section{TYPE IIA FLUX MODELS}

We will present two kinds of models where some of the fluxes are free parameters and can be very large. In the first kind of models, the very large fluxes that we introduce do not change the RR tadpole cancellation conditions. While in the second kind of models, the very large fluxes do contribute to one of the D-brane RR tadpole cancellation conditions. 


\section{A. Model TIIA-I}

In all the Pati-Salam models, $S U(5)$ models, and flipped $S U(5)$ models that have been constructed previously [38, 39], the fluxes $h_{0}, m, q$, and $a$ are not fixed. And only one quadratic combination of them $\left(h_{0} m+3 q a\right)$ is determined by the RR tadpole cancellation conditions. Thus, three of the four fluxes $h_{0}, m, q$, and $a$ can be free parameters. Moreover, $e$ and $e_{0}$ can also be taken as free parameters. As an example, let us study a concrete model which is model TI-U-4 in Ref. [38]. We present the D-brane configurations and intersection numbers in Table II, and the particle spectrum in the observed sector in Tables III and IV. The phenomenological consequences have been discussed in Ref. [38]. Similar to the discussions in Ref. [17, 18], we can explain the SM fermion masses and mixings because all the SM fermions and Higgs fields arise from the intersections on the third torus.

\begin{tabular}{|c|c|c|c|c|c|c|c|c|c|c|c|c|c|}
\hline stack & $N$ & $\left(n_{1}, l_{1}\right)\left(n_{2}, l_{2}\right)\left(n_{3}, l_{3}\right)$ & A & $\mathrm{S}$ & $b$ & $b^{\prime}$ & $c$ & $c^{\prime}$ & $d$ & $d^{\prime}$ & $e$ & $e^{\prime}$ & O6 \\
\hline$a$ & 4 & $(0,-1)(1,1)(3,1)$ & 1 & -1 & 30 & (1) & & $(3)$ & 2 & $0(2)$ & -3 & - & 1 \\
\hline$b$ & 2 & $(-1,-1)(2,0)(-3,1)$ & 0 & 0 & - & - & $6 \mid 0$ & (3) & -1 & -5 & 6 & - & $0(1)$ \\
\hline$c$ & 2 & $(1,-1)(-1,1)(0,-2)$ & -2 & 2 & - & - & - & - & $0(10)$ & 2 & $0(1)$ & - & -2 \\
\hline$d$ & 2 & $(2,3)(1,-1)(2,0)$ & 0 & 0 & - & - & - & - & - & - & 6 & - & $0(3)$ \\
\hline$e$ & 1 & $(1,0)(0,-2)(0,2)$ & 0 & 0 & - & - & - & - & - & - & - & - & (4) \\
\hline O6 & 5 & $(1,0)(2,0)(2,0)$ & & 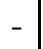 & & 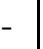 & & 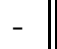 & 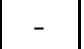 & - & - & 1 & \\
\hline
\end{tabular}

TABLE II: D6-brane configurations and intersection numbers for Model TIIA-I on Type IIA $\mathbf{T}^{\mathbf{6}}$ orientifold. The complete gauge symmetry is $\left[U(4)_{C} \times U(2)_{L} \times U(2)_{R}\right]_{\text {observable }} \times[U(2) \times U S p(2) \times$ USp(10)] hidden, the SM fermions and Higgs fields arise from the intersections on the third twotorus, and the complex structure parameters are $6 \chi_{1}=2 \chi_{2}=\chi_{3}=2 \sqrt{6}$. To satisfy the RR tadpole cancellation conditions, we choose $h_{0} m+3 a q=-48$.

Moreover, we can calculate the real parts of dilaton and complex structure moduli

$$
\begin{aligned}
& \operatorname{Re} s=\frac{6^{3 / 4} e^{-\phi_{4}}}{24 \pi}, \operatorname{Re} u_{1}=\frac{6^{3 / 4} e^{-\phi_{4}}}{2 \pi}, \\
& \operatorname{Re} u_{2}=\frac{6^{3 / 4} e^{-\phi_{4}}}{6 \pi}, \operatorname{Re} u_{3}=\frac{6^{3 / 4} e^{-\phi_{4}}}{12 \pi} .
\end{aligned}
$$

And then, we obtain the gauge couplings at the string scale in the observable sector

$$
2 g_{a}^{-2}=g_{b}^{-2}=g_{c}^{-2}=5 \frac{6^{3 / 4} e^{-\phi_{4}}}{12 \pi} .
$$

So, the gauge couplings for $S U(2)_{L}$ and $S U(2)_{R}$ are unified at the string scale, and are half of these for $S U(3)_{C}$ and $U(1)_{B-L}$. 


\begin{tabular}{|c||c||c|c|c|}
\hline Representation & Multiplicity & $U(1)_{a}$ & $U(1)_{b}$ & $U(1)_{c}$ \\
\hline \hline$\left(4_{a}, \overline{2}_{b}\right)$ & 3 & 1 & -1 & 0 \\
$\left(\overline{4}_{a}, 2_{c}\right)$ & 3 & -1 & 0 & 1 \\
$\left(2_{b}, \overline{2}_{c}\right)$ & 6 & 0 & 1 & -1 \\
\hline$\left(4_{a}, 2_{c}\right)$ & 3 & 1 & 0 & 1 \\
$\left(\overline{4}_{a}, \overline{2}_{c}\right)$ & 3 & -1 & 0 & -1 \\
\hline \hline $6_{a}$ & 1 & 2 & 0 & 0 \\
$\overline{10}_{a}$ & 1 & -2 & 0 & 0 \\
$1_{c}$ & 2 & 0 & 0 & -2 \\
$3_{c}$ & 2 & 0 & 0 & 2 \\
\hline
\end{tabular}

TABLE III: The particle spectrum in observable sector in Model TIIA-I with gauge symmetry $\left[U(4)_{C} \times U(2)_{L} \times U(2)_{R}\right]_{\text {observable }} \times[U(2) \times U S p(2) \times U S p(10)]_{\text {hidden }}$. Here, $a, b$ and $c$ denote the gauge groups $U(4)_{C}, U(2)_{L}$ and $U(2)_{R}$, respectively.

Next, we shall study the flux parameter spaces when the fluxes $h_{0}, m, q$, and/or $a$ are very large. The general solution to $h_{0} m+3 q a=-48$ is

$$
m=2 N_{1}, \quad h_{0}=-24 N_{2}, \quad q=2 N_{3}, \quad a=\frac{8\left(N_{1} N_{2}-1\right)}{N_{3}},
$$

where $\left(N_{1} N_{2}-1\right)$ must be the multiple of $N_{3}$. For example, we can have $N_{3}= \pm 1$ or $N_{3}= \pm\left(N_{1} N_{2}-1\right)$. If $a$ is very large, the theory will be strong coupled, and the magnitude of the cosmological constant is very large. Although we can make the theory to be weakly coupled and the magnitude of the cosmological constant to be small by introducing the very large fluxes $e$ and/or $e_{0}$, we do not consider the case with large $a$ for simplicity. And then we obtain $N_{3}= \pm\left(N_{1} N_{2}-1\right)$. To be concrete, we choose that $N_{1}$ and $N_{2}$ are positive integers, and $N_{3}=\left(N_{1} N_{2}-1\right)$. For simplicity, we assume $e_{0}=e=0$.

In the very large $N_{1}$ and/or $N_{2}$ limit, we obtain

$$
\lambda_{0}=1-\frac{1}{N_{1} N_{2}} \longrightarrow 1, \quad \lambda \simeq \frac{10^{2 / 3}}{20} .
$$

In addition, we obtain

$$
\begin{gathered}
\operatorname{Re} s \sim N_{1} N_{2}^{2}, \quad \text { Ret } \sim N_{2}, \quad \operatorname{Re} u_{i} \sim N_{1} N_{2}^{2}, \\
e^{-\phi_{4}} \sim N_{1} N_{2}^{2}, \quad e^{-\phi} \sim N_{1} N_{2}^{1 / 2}, \quad g_{a, b, c}^{-2} \sim N_{1} N_{2}^{2}, \\
V_{0} \sim N_{1}^{-2} N_{2}^{-5} .
\end{gathered}
$$




\begin{tabular}{|c||c||c|c|c|c|}
\hline Representation & Multiplicity & $U(1)_{a}$ & $U(1)_{b}$ & $U(1)_{c}$ & $U(1)_{d}$ \\
\hline \hline$\left(4_{a}, \overline{2}_{d}\right)$ & 2 & 1 & 0 & 0 & -1 \\
$\left(\overline{4}_{a}, 2_{e}\right)$ & 3 & -1 & 0 & 0 & 0 \\
$\left(4_{a}, 1_{O 6}\right)$ & 1 & 1 & 0 & 0 & 0 \\
$\left(\overline{2}_{b}, 2_{d}\right)$ & 1 & 0 & -1 & 0 & 1 \\
$\left(\overline{2}_{b}, \overline{2}_{d}\right)$ & 5 & 0 & -1 & 0 & -1 \\
$\left(2_{b}, 2_{e}\right)$ & 6 & 0 & 1 & 0 & 0 \\
$\left(2_{c}, 2_{d}\right)$ & 2 & 0 & 0 & 1 & 1 \\
$\left(\overline{2}_{c}, 10_{O 6}\right)$ & 2 & 0 & 0 & -1 & 0 \\
$\left(2_{d}, 2_{e}\right)$ & 6 & 0 & 0 & 0 & 1 \\
\hline
\end{tabular}

TABLE IV: The exotic particle spectrum in Model TIIA-I with gauge symmetry $\left[U(4)_{C} \times U(2)_{L} \times\right.$ $\left.U(2)_{R}\right]_{\text {observable }} \times[U(2) \times U S p(2) \times U S p(10)]_{\text {hidden }}$. Here, $a, b, c, d, e$ and $O 6$ denote the gauge groups $U(4)_{C}, U(2)_{L}, U(2)_{R}, U(2), U S p(2)$ and $U S p(10)$, respectively.

Thus, in the very large $N_{1}$ and/or $N_{2}$ limit, the theory will be very weakly coupled, the magnitude of the cosmological constant will be close to zero, and then the AdS vacua approach to the Minkowski vacua.

\section{B. Model TIIA-II}

In the above kind of models, we consider the scenario where the fluxes can be very large while $\left(h_{0} m+3 q a\right)$ is a negative constant. In this subsection, we consider the scenario where the fluxes can be very large, and $\left(h_{0} m+3 q a\right)$ is also a large negative number. If $\left(h_{0} m+3 q a\right)$ is negative and very large, we can alaways satisfy the RR tadpole cancellation conditions by introducing enough D-branes. However, we will have many chiral exotic particles that can not be decoupled easily. Thus, we consider a special scenario where the complex structure parameters $\chi_{i}$ are also very large, and we keep $h_{0} /\left(\chi_{i} \chi_{j}\right)$ and $a /\left(\chi_{i} \chi_{j}\right)$ constant. Then the very large fluxes only affect the first $R R$ tadpole cancellation condition in Eq. (37).

We present the D-brane configurations and intersection numbers in Table $\mathrm{V}$, and the particle spectrum in the observed sector in Table VI. In addition, we choose

$$
\begin{gathered}
\chi_{1}=\frac{\sqrt{\kappa}}{3 \sqrt{2}}, \quad \chi_{2}=\chi_{3}=\sqrt{2 \kappa}, \\
h_{0} m+3 q a=-8 \kappa
\end{gathered}
$$




\begin{tabular}{|c|c|c|c|c|c|c|c|c|c|c|c|}
\hline stk & $N$ & $\left(n_{1}, l_{1}\right)\left(n_{2}, l_{2}\right)\left(n_{3}, l_{3}\right)$ & $\mathrm{A} \mid \mathrm{S}$ & $b$ & $b^{\prime}$ & $c$ & $c^{\prime}$ & $d$ & $e$ & $f$ & $O 6$ \\
\hline$a$ & 4 & $((1,0) \quad(-1,-1)(-1,1)$ & 0 & 3 & $0(3$ & & $(3)$ & $0(1)$ & 1 & -1 & $0(1)$ \\
\hline$b$ & 2 & $(-1,3)(-2,0)(1,1)$ & \begin{tabular}{l|l}
0 & 0 \\
\end{tabular} & ) & - & & $(1)$ & -6 & $0(1)$ & 2 & $4(3)$ \\
\hline$c$ & 2 & $(1,3)(-1,1)(-2,0)$ & 00 & $\mid-$ & - & - & - & 6 & -2 & $0(1)$ & \\
\hline$d$ & 1 & $(1,0) \quad(0,-2)(0,2)$ & 0 & ) & - & & - & - & $0(2$ & 0( & $0(4)$ \\
\hline$e$ & 3 & $(0,-1)(2,0)(0,2)$ & 00 & ) & - & - & - & - & - & $0(4)$ & \\
\hline$f$ & 3 & $(0,1)(0,2)(2,0)$ & 0 & & - & - & - & - & - & - & $0(2)$ \\
\hline$O 6$ & $+\kappa)$ & $(1,0)(2,0)(2,0)$ & & & & & & & & & \\
\hline
\end{tabular}

TABLE V: D6-brane configurations and intersection numbers for Model TIIA-II on Type IIA $\mathbf{T}^{\mathbf{6}}$ orientifold. The complete gauge symmetry is $U(4)_{C} \times U(2)_{L} \times U(2)_{R} \times U S p(2) \times U S p(6)^{2} \times$ $U S p(2(1+\kappa))$, and the SM fermions and Higgs fields arise from the intersections on the first two-torus.

where $\kappa=1,2,3, \cdots$. From Eqs. (33), (35) and (36), we obtain the generic solution to the flux consistent equations:

$$
\begin{gathered}
h_{0}=4 \kappa \eta, \quad m=-4 \eta, \quad q=2 \eta^{\prime}, \quad a=4 \kappa \eta^{\prime}, \\
b_{1}=6 \eta^{\prime}, \quad b_{2}=b_{3}=36 \eta^{\prime}, \quad h_{1}=-2 \eta, \quad h_{2}=h_{3}=-12 \eta,
\end{gathered}
$$

where $\eta= \pm 1$ and $\eta^{\prime}= \pm 1$. In this case, $\kappa$ can be considered as a scale factor. Then in the very large $\kappa$ limit, we obtain

$$
\begin{gathered}
\operatorname{Re} s \longrightarrow \kappa^{-1} \operatorname{Re} s, \operatorname{Re} t \longrightarrow \operatorname{Re} t, \operatorname{Re} u_{i} \longrightarrow \operatorname{Re} u_{i}, \\
\operatorname{Im} s \longrightarrow \operatorname{Im} s, \operatorname{Im} t \longrightarrow \operatorname{Im} t, \operatorname{Im} u_{i} \longrightarrow \operatorname{Im} u_{i}, \\
e^{-\phi_{4}} \longrightarrow \kappa^{-1 / 4} e^{-\phi_{4}}, e^{-\phi} \longrightarrow \kappa^{-1 / 4} e^{-\phi}, V_{0} \longrightarrow \kappa V_{0} .
\end{gathered}
$$

Thus, the gauge theory will not become strong coupled and the gauge couplings approach to the fixed constants. However, the magnitude of the cosmological constant will be very large. In order to have small magnitude for the cosmological constant, we can introduce very large $e_{0}$ and/or $e$, as in the last Section.

Next, let us consider the phenomenological consequences. The anomalies from three global $U(1)$ s of $U(4)_{C}, U(2)_{L}$ and $U(2)_{R}$ are cancelled by the Green-Schwarz mechanism, and the gauge fields of these $U(1)$ s obtain masses via the linear $B \wedge F$ couplings. So, 


\begin{tabular}{|c||c||c|c|c|c|}
\hline Representation & Multiplicity & $U(1)_{a}$ & $U(1)_{b}$ & $U(1)_{c}$ & Field \\
\hline \hline$\left(4_{a}, \overline{2}_{b}\right)$ & 3 & 1 & -1 & 0 & $F_{L}\left(Q_{L}, L_{L}\right)$ \\
$\left(\overline{4}_{a}, 2_{c}\right)$ & 3 & -1 & 0 & 1 & $F_{R}\left(Q_{R}, L_{R}\right)$ \\
$\left(2_{b}, \overline{2}_{c}\right)$ & 6 & 0 & 1 & -1 & $\Phi_{i}\left(H_{u}^{i}, H_{d}^{i}\right)$ \\
\hline$\left(4_{a}, 2_{c}\right)$ & 3 & 1 & 0 & 1 & \\
$\left(\overline{4}_{a}, \overline{2}_{c}\right)$ & 3 & -1 & 0 & -1 & \\
\hline \hline$\left(4_{a}, 6_{e}\right)$ & 1 & 1 & 0 & 0 & $X_{a e}$ \\
$\left(4_{a}, 6_{f}\right)$ & 1 & -1 & 0 & 0 & $X_{a f}$ \\
$\left(2_{b}, 2_{d}\right)$ & 6 & 0 & -1 & 0 & $X_{b d}^{i}$ \\
$\left(2_{b}, 6_{f}\right)$ & 2 & 0 & 1 & 0 & $X_{b f}^{i}$ \\
$\left(2_{c}, 2_{d}\right)$ & 6 & 0 & 0 & 1 & $X_{c d}^{i}$ \\
$\left(2_{c}, 6_{e}\right)$ & 2 & 0 & 0 & -1 & $X_{c e}^{i}$ \\
\hline
\end{tabular}

TABLE VI: The particle spectrum in observable sector in Model TIIA-II with gauge symmetry $\left[U(4)_{C} \times U(2)_{L} \times U(2)_{R}\right]_{\text {observable }} \times\left[U S p(2) \times U S p(6)^{2} \times U S p(2+2 \kappa)\right]_{\text {hidden }}$. Here, $a, b$ and $c$ denote the gauge groups $U(4)_{C}, U(2)_{L}$ and $U(2)_{R}$, respectively.

the effective gauge symmetry is $S U(4)_{C} \times S U(2)_{L} \times S U(2)_{R}$. In order to break the gauge symmetry, on the first two-torus, we split the $a$ stack of D-branes into $a_{1}$ and $a_{2}$ stacks with 3 and 1 D-branes, respectively, and split the $c$ stack of D-branes into $c_{1}$ and $c_{2}$ stacks with 1 D-brane for each one. Then, the gauge symmetry is further broken down to $S U(3)_{C} \times$ $S U(2)_{L} \times U(1)_{I_{3 R}} \times U(1)_{B-L}$. We can break the $U(1)_{I_{3 R}} \times U(1)_{B-L}$ gauge symmetry down to the $U(1)_{Y}$ gauge symmetry by giving vacuum expectation values (VEVs) to the vectorlike particles with quantum numbers $(\mathbf{1}, \mathbf{1}, \mathbf{1} / \mathbf{2}, \mathbf{- 1})$ and $(\mathbf{1}, \mathbf{1}, \mathbf{- 1} / \mathbf{2}, \mathbf{1})$ under $S U(3)_{C} \times$ $S U(2)_{L} \times U(1)_{I_{3 R}} \times U(1)_{B-L}$ from $a_{2} c_{1}^{\prime}$ D-brane intersections. Similar to the discussions in Ref. [17, 18], we can explain the SM fermion masses and mixings because all the SM fermions and Higgs fields arise from the intersections on the first two-torus.

To decouple the chiral exotic particles, we assume that the $e$ and $f$ stacks of the D-branes are on the top of each other on the first two-torus, the $d$ and $f$ stacks of the D-branes are on the top of each other on the second two-torus, and the $d$ and $e$ stacks of the D-branes are on the top of each other on the third two-torus. Then we have the vector-like particles $\left(X_{e f}^{i}\right.$ and $\left.\bar{X}_{e f}^{i}\right),\left(X_{d f}^{j}\right.$ and $\left.\bar{X}_{d f}^{j}\right)$, and $\left(X_{d e}^{j}\right.$ and $\left.\bar{X}_{d e}^{j}\right)$ whose quantum numbers are $\left(6_{e}, 6_{f}\right),\left(2_{d}, 6_{f}\right)$, and $\left(2_{d}, 6_{e}\right)$, respectively, where $i=1,2,3,4$ and $j=1,2$. Then, we have the following superpotential

$$
W \supset X_{a e} X_{a f}\left(X_{e f}^{i}+\bar{X}_{e f}^{i}\right)+X_{b d}^{i} X_{b f}^{j}\left(X_{d f}^{k}+\bar{X}_{d f}^{k}\right)+X_{c d}^{i} X_{c e}^{j}\left(X_{d e}^{k}+\bar{X}_{d e}^{k}\right)
$$


where we neglect the Yukawa couplings. Thus, if $\left(X_{e f}^{i}\right.$ and $\left.\bar{X}_{e f}^{i}\right),\left(X_{d f}^{j}\right.$ and $\left.\bar{X}_{d f}^{j}\right)$, and $\left(X_{d e}^{j}\right.$ and $\bar{X}_{d e}^{j}$ ) obtain VEVs, we might decouple all the chiral exotic particles. In short, below the string scale, we may just have the supersymmetric Standard Model.

Moreover, we can calculate the real parts of the dilaton and complex structure moduli

$$
\begin{aligned}
& \operatorname{Re} s=\frac{\sqrt{6 \sqrt{2}} e^{-\phi_{4}}}{4 \pi \kappa^{3 / 4}}, \operatorname{Re} u_{1}=\frac{\sqrt{6 \sqrt{2}} \kappa^{1 / 4} e^{-\phi_{4}}}{2 \pi}, \\
& \operatorname{Re} u_{2}=\frac{\sqrt{6 \sqrt{2}} \kappa^{1 / 4} e^{-\phi_{4}}}{12 \pi}, \operatorname{Re} u_{3}=\frac{\sqrt{6 \sqrt{2}} \kappa^{1 / 4} e^{-\phi_{4}}}{12 \pi} .
\end{aligned}
$$

And then, we obtain the gauge couplings at the string scale in the observable sector

$$
2 g_{a}^{-2}=g_{b}^{-2}=g_{c}^{-2}=\frac{\sqrt{6 \sqrt{2}} \kappa^{1 / 4} e^{-\phi_{4}}}{2 \pi}\left(\frac{1}{\kappa}+\frac{1}{2}\right) \text {. }
$$

So, the gauge couplings for $S U(2)_{L}$ and $S U(2)_{R}$ are unified at the string scale, and are half of these for $S U(3)_{C}$ and $U(1)_{B-L}$.

\section{FLUX MODEL BUILDING ON TYPE IIB ORIENTIFOLD}

We consider the Type IIB string theory compactified on a $\mathbf{T}^{\mathbf{6}}$ orientifold where $\mathbf{T}^{\mathbf{6}}$ is a six-torus factorized as $\mathbf{T}^{\mathbf{6}}=\mathbf{T}^{\mathbf{2}} \times \mathbf{T}^{\mathbf{2}} \times \mathbf{T}^{\mathbf{2}}$ whose complex coordinates are $z_{i}, i=1,2,3$ for the $i$-th two-torus, respectively $[24,25,28]$. The orientifold projection is implemented by gauging the symmetry $\Omega R$, where $\Omega$ is world-sheet parity, and $R$ is given by

$$
R:\left(z_{1}, z_{2}, z_{3}\right) \rightarrow\left(-z_{1},-z_{2},-z_{3}\right)
$$

Thus, the model contains 64 O3-planes. In order to cancel the negative RR charges from these O3-planes, we introduce the magnetized $\mathrm{D}(3+2 \mathrm{n})$-branes which are filling up the fourdimensional Minkowski space-time and wrapping 2n-cycles on the compact manifold. Concretely, for one stack of $N_{a}$ D-branes wrapped $m_{a}^{i}$ times on the $i$-th two-torus $\mathbf{T}_{\mathbf{i}}^{2}$, we turn on $n_{a}^{i}$ units of magnetic fluxes $F_{a}^{i}$ for the center of mass $U(1)_{a}$ gauge factor on $\mathbf{T}_{\mathbf{i}}^{\mathbf{2}}$, such that

$$
m_{a}^{i} \frac{1}{2 \pi} \int_{T_{i}^{2}} F_{a}^{i}=n_{a}^{i}
$$

where $m_{a}^{i}$ can be half integer for tilted two-torus. Then, the D9-, D7-, D5- and D3-branes contain $0,1,2$ and 3 vanishing $m_{a}^{i}$ s, respectively. Introducing for the $i$-th two-torus the even homology classes $\left[\mathbf{0}_{i}\right]$ and $\left[\mathbf{T}_{i}^{2}\right]$ for the point and two-torus, respectively, the vectors of the $\mathrm{RR}$ charges of the $a$ stack of D-branes and its image are

$$
\begin{aligned}
& {\left[\Pi_{a}\right]=\prod_{i=1}^{3}\left(n_{a}^{i}\left[\mathbf{0}_{i}\right]+m_{a}^{i}\left[\mathbf{T}_{i}^{2}\right]\right),} \\
& {\left[\Pi_{a}^{\prime}\right]=\prod_{i=1}^{3}\left(n_{a}^{i}\left[\mathbf{0}_{i}\right]-m_{a}^{i}\left[\mathbf{T}_{i}^{2}\right]\right)}
\end{aligned}
$$


TABLE VII: General spectrum for magnetized D-branes on the Type IIB $\mathbf{T}^{\mathbf{6}}$ orientifold.

\begin{tabular}{|c|c|}
\hline Sector & Representation \\
\hline \hline$a a$ & $U\left(N_{a}\right)$ vector multiplet \\
& 3 adjoint multiplets \\
\hline$a b+b a$ & $I_{a b}\left(N_{a}, \bar{N}_{b}\right)$ multiplets \\
\hline$a b^{\prime}+b^{\prime} a$ & $I_{a b^{\prime}}\left(N_{a}, N_{b}\right)$ multiplets \\
\hline$a a^{\prime}+a^{\prime} a$ & $\frac{1}{2}\left(I_{a a^{\prime}}-I_{a O 3}\right) \quad$ symmetric multiplets \\
& $\frac{1}{2}\left(I_{a a^{\prime}}+I_{a O 3}\right) \quad$ anti-symmetric multiplets \\
\hline
\end{tabular}

respectively. The "intersection numbers" in Type IIA language, which determine the chiral massless spectrum, are

$$
I_{a b}=\left[\Pi_{a}\right] \cdot\left[\Pi_{b}\right]=\prod_{i=1}^{3}\left(n_{a}^{i} m_{b}^{i}-n_{b}^{i} m_{a}^{i}\right) .
$$

Moreover, for a stack of $N \mathrm{D}(2 \mathrm{n}+3)$-branes whose homology classes on $\mathbf{T}^{\mathbf{6}}$ is (not) invariant under $\Omega R$, we obtain a $(U(N)) U S p(2 N)$ gauge symmetry with three (adjoint) anti-symmetric chiral superfields due to the orbifold projection. The physical spectrum is presented in Table VII.

The flux models on Type IIB orientifolds with four-dimensional $N=1$ supersymmetry are primarily constrained by the $\mathrm{RR}$ tadpole cancellation conditions that will be given later, the four-dimensional $N=1$ supersymmetry condition, and the K-theory anomaly free conditions. For the D-branes with world-volume magnetic field $F_{a}^{i}=n_{a}^{i} /\left(m_{a}^{i} \chi_{i}\right)$ where $\chi_{i}$ is the area of $\mathbf{T}_{\mathbf{i}}^{\mathbf{2}}$ in string units, the condition to preserve the four-dimensional $N=1$ supersymmetry is [28]

$$
\sum_{i}\left(\tan ^{-1}\left(F_{a}^{i}\right)^{-1}+\theta\left(n_{a}^{i}\right) \pi\right)=0 \bmod 2 \pi,
$$

where $\theta\left(n_{a}^{i}\right)=1$ for $n_{a}^{i}<0$ and $\theta\left(n_{a}^{i}\right)=0$ for $n_{a}^{i} \geq 0$. The K-theory anomaly free conditions are

$$
\begin{aligned}
& \sum_{a} N_{a} m_{a}^{1} m_{a}^{2} m_{a}^{3}=\sum_{a} N_{a} m_{a}^{1} n_{a}^{2} n_{a}^{3}=\sum_{a} N_{a} n_{a}^{1} m_{a}^{2} n_{a}^{3} \\
& =\sum_{a} N_{a} n_{a}^{1} n_{a}^{2} m_{a}^{3}=0 \quad \bmod 2 .
\end{aligned}
$$


And the holomorphic gauge kinetic function for a generic stack of $\mathrm{D}(2 \mathrm{n}+3)$-branes is given by [48, 49]

$$
\begin{aligned}
f_{a}= & \frac{1}{\kappa_{a}}\left(n_{a}^{1} n_{a}^{2} n_{a}^{3} s-n_{a}^{1} m_{a}^{2} m_{a}^{3} t_{1}\right. \\
& \left.-n_{a}^{2} m_{a}^{1} m_{a}^{3} t_{2}-n_{a}^{3} m_{a}^{1} m_{a}^{2} t_{3}\right),
\end{aligned}
$$

where $\kappa_{a}$ is equal to 1 and 2 for $U(n)$ and $U S p(2 n)$, respectively.

We turn on the NSNS flux $h_{0}$, RR flux $e_{i}$, non-geometric fluxes $b_{i i}$ and $\bar{b}_{i i}$, and the S-dual fluxes $f_{i}, g_{i j}$ and $g_{i i}[32,34]$. To avoid the subtleties, these fluxes should be even integers due to the Dirac quantization. For simplicity, we assume

$$
\begin{aligned}
& e_{i}=e, \quad b_{i i}=\beta, \quad \bar{b}_{i i}=\bar{\beta}, \\
& f_{i}=f, g_{i j}=-g_{i i}=g,
\end{aligned}
$$

where $i \neq j$. Then the constraint on fluxes from Bianchi indetities is

$$
f \bar{\beta}=g \beta .
$$

The RR tadpole cancellation conditions are

$$
\begin{aligned}
& \sum_{a} N_{a} n_{a}^{1} n_{a}^{2} n_{a}^{3}=16, \\
& \sum_{a} N_{a} n_{a}^{i} m_{a}^{j} m_{a}^{k}=-\frac{1}{2} e \bar{\beta}, \\
& N_{\mathrm{NS}_{i}}=0, \quad N_{\mathrm{I}_{i}}=0,
\end{aligned}
$$

where $i \neq j \neq k \neq i$, and the $N_{\mathrm{NS} 7_{i}}$ and $N_{\mathrm{I} 7_{i}}$ denote the NS 7-brane charge and the other 7-brane charge, respectively [32, 34]. Thus, if $e \bar{\beta}<0$, the $\mathrm{RR}$ tadpole cancellation conditions are relaxed elegantly because $-e \bar{\beta} / 2$ only needs to be even integer. Moreover, we have 7 moduli fields in the supergravity theory basis, the dilaton $s$, three Kähler moduli $t_{i}$, and three complex structure moduli $u_{i}$. With the above fluxes, we can assume

$$
t \equiv t_{1}+t_{2}+t_{3}, \quad u_{1}=u_{2}=u_{3} \equiv u
$$

Then the superpotential becomes [34]

$$
\mathcal{W}=3 i e u+i h_{0} s-t\left(\beta u-i \bar{\beta} u^{2}\right)-s t(f-i g u)
$$

The Kähler potential for these moduli is

$$
\mathcal{K}=-\ln (s+\bar{s})-\sum_{i=1}^{3} \ln \left(t_{i}+\bar{t}_{i}\right)-\sum_{i=1}^{3} \ln \left(u_{i}+\bar{u}_{i}\right)
$$


Thus, for the supersymmetric Minkowski vacua, we have

$$
\mathcal{W}=\partial_{s} \mathcal{W}=\partial_{t} \mathcal{W}=\partial_{u} \mathcal{W}=0
$$

From $\partial_{s} \mathcal{W}=\partial_{t} \mathcal{W}=0$, we obtain [34]

$$
t=\frac{i h_{0}}{f-i g u}, \quad s=-\frac{\beta}{f} u
$$

then the superpotential turns out

$$
\mathcal{W}=\left(3 e-\frac{h_{0} \beta}{f}\right) i u .
$$

Therefore, to satisfy $\mathcal{W}=\partial_{u} \mathcal{W}=0$, we obtain [34]

$$
3 e f=\beta h_{0} .
$$

Because $\operatorname{Re} s>0, \operatorname{Ret}_{i}>0$ and $\operatorname{Re} u_{i}>0$, we require

$$
\frac{h_{0}}{g}<0, \quad \frac{\beta}{f}<0 \text {. }
$$

In general, this kind of D-brane models might have the Freed-Witten anomalies [24, 47]. Interestingly, the Freed-Witten anomalies can be cancelled by introducing additional Dbranes [24]. In particular, the additional D-branes will not affect the main properties of the D-brane models, for example, the four-dimensional $N=1$ supersymmetry and the chiral spectra, etc [24]. Therefore, we can construct this kind of D-brane models by ignoring the subtlety of the Freed-Witten anomalies.

\section{TYPE IIB FLUX MODELS}

Choosing $e \bar{\beta}=-12 \kappa$ where $\kappa=1,2,3, \ldots$, we construct a series of realistic Pati-Salam models with gauge symmetry $U(4)_{C} \times U(2)_{L} \times U(2)_{R} \times U S p(10) \times U S p(6(\kappa-1))^{3}$. We present their D-brane configurations and intersection numbers in Table VIII, and the resulting spectra in Tables $[\mathrm{IX}$ and $\mathrm{X}$. Table $[\mathrm{IX}$ is the spectra for $\kappa=1$ while Table $\mathrm{X}$ is the additional spectra due to $\kappa>1$.

The anomalies from three global $U(1) \mathrm{s}$ of $U(4)_{C}, U(2)_{L}$ and $U(2)_{R}$ are cancelled by the Green-Schwarz mechanism, and the gauge fields of these $U(1)$ s obtain masses via the linear $B \wedge F$ couplings. So, the effective gauge symmetry is $S U(4)_{C} \times S U(2)_{L} \times S U(2)_{R}$. In order to break the gauge symmetry, on the first two-torus, we split the $a$ stack of D-branes into $a_{1}$ and $a_{2}$ stacks with 3 and 1 D-branes, respectively, and split the $c$ stack of D-branes into $c_{1}$ and $c_{2}$ stacks with $1 \mathrm{D}$-brane for each one. Then, the gauge symmetry is further broken down to 
$S U(3)_{C} \times S U(2)_{L} \times U(1)_{I_{3 R}} \times U(1)_{B-L}$. We can break the $U(1)_{I_{3 R}} \times U(1)_{B-L}$ gauge symmetry down to the $U(1)_{Y}$ gauge symmetry by giving VEVs to the vector-like particles with quantum numbers $(\mathbf{1}, \mathbf{1}, \mathbf{1} / \mathbf{2}, \mathbf{- 1})$ and $(\mathbf{1}, \mathbf{1}, \mathbf{- 1} / \mathbf{2}, \mathbf{1})$ under $S U(3)_{C} \times S U(2)_{L} \times U(1)_{I_{3 R}} \times U(1)_{B-L}$ from $a_{2} c_{1}^{\prime}$ D-brane intersections. Similar to the discussions in Ref. [17, 18], we can explain the SM fermion masses and mixings via the Higgs fields $H_{u}^{i}, H_{u}^{\prime}, H_{d}^{i}$ and $H_{d}^{\prime}$ because all the SM fermions and Higgs fields arise from the intersections on the first two-torus.

First, let us consider $\kappa=1$ case. Similar to the discussions in Ref. [34], we can decouple the chiral exotic particles in Table $\llbracket \mathrm{IX}$ as following. We assume that the $T_{R}^{i}$ and $S_{R}^{i}$ obtain VEVs at about the string scale, and their VEVs satisfy the D-flatness of $U(1)_{R}$. The chiral exotic particles can obtain masses via the following superpotential

$$
W \supset \frac{1}{M_{\mathrm{St}}} S_{R}^{i} S_{R}^{j} T_{R}^{k} T_{R}^{l}+T_{R}^{i} X^{j} X^{k},
$$

where $M_{\mathrm{St}}$ is the string scale, and we neglect the order $1(\mathcal{O}(1))$ coefficients. In addition, the vector-like particles $S_{L}^{i}$ and $\bar{S}_{L}^{i}$ in the anti-symmetric representation of $S U(2)_{L}$ can obtain VEVs close to the string scale while keeping the D-flatness of $U(1)_{L}$. Thus, we can decouple all the Higgs bidoublets close to the string scale except one pair of the linear combinations of the Higgs doublets for the electroweak symmetry breaking at the low energy by fine-tuning the following superpotential

$$
\begin{aligned}
W \supset & \Phi_{i}\left(\bar{S}_{L}^{j} \Phi^{\prime}+S_{R}^{j} \bar{\Phi}^{\prime}\right)+\bar{\Phi}_{i}\left(T_{R}^{j} \Phi^{\prime}+S_{L}^{j} \bar{\Phi}^{\prime}\right) \\
& +\frac{1}{M_{\mathrm{St}}}\left(\bar{S}_{L}^{i} S_{R}^{j} \Phi_{k} \Phi_{l}+S_{L}^{i} T_{R}^{j} \bar{\Phi}_{k} \bar{\Phi}_{l}\right. \\
& \left.+\bar{S}_{L}^{i} T_{R}^{j} \Phi^{\prime} \Phi^{\prime}+S_{L}^{i} S_{R}^{j} \bar{\Phi}^{\prime} \bar{\Phi}^{\prime}\right)
\end{aligned}
$$

In short, below the string scale for $\kappa=1$, we have the supersymmetric SMs which may have zero, one, or a few SM singlets from $S_{L}^{i}, \bar{S}_{L}^{i}$, and/or $S_{R}^{i}$. And then the low bound on the lightest CP-even Higgs boson mass in the minimal supersymmetric SM can be relaxed if we have the SM singlet(s) at low energy [50].

Second, we consider the case with $\kappa>1$, and decouple the additional chiral exotic superfields in Table $\mathrm{X}$. We assume that the $e$ and $f$ stacks of the D-branes are on the top of each other on the first two-torus. Then we have the vector-like particles $X_{e f}^{i}$ and $\bar{X}_{e f}^{i}$ whose quantum numbers are $\left((6(\kappa-1))_{e},(6(\kappa-1))_{f}\right)$ under the $U S p(6(\kappa-1))_{e} \times U S p(6(\kappa-1))_{f}$ gauge symmetry where $i=1,2$. Also, we assume that $X_{e f}^{i}$ and $\bar{X}_{e f}^{i}$ obtain VEVs. Then, we have the following superpotential

$$
W \supset\left(X_{e f}^{i}+\bar{X}_{e f}^{i}\right) X_{a e} X_{a f}+S_{L}^{i} X_{b d}^{j} X_{b d}^{j}+\bar{S}_{L}^{i} X_{b e} X_{b e}+S_{R}^{i} X_{c f} X_{c f} .
$$

Thus, we can decouple the extra chiral exotic particles due to $\kappa>1$ as well. In short, below the string scale, we also have the supersymmetric SMs with or without additional SM singlets. 


\begin{tabular}{|c|c||c||c|c||c|c|c|c||c|c|c|c|}
\hline stk & $N$ & $\left(n_{1}, l_{1}\right)\left(n_{2}, l_{2}\right)\left(n_{3}, l_{3}\right)$ & $\mathrm{A}$ & $\mathrm{S}$ & $b$ & $b^{\prime}$ & $c$ & $c^{\prime}$ & $d$ & $e$ & $f$ & $O 3$ \\
\hline \hline$a$ & 4 & $(1,0)(1,-1)(1,1)$ & 0 & 0 & 3 & $0(3)$ & -3 & $0(3)$ & $0(1)$ & -1 & 1 & $0(1)$ \\
\hline$b$ & 2 & $(1,-3)(1,1)(1,0)$ & 0 & 0 & - & - & $0(6)$ & $0(1)$ & -3 & 1 & $0(1)$ & $0(3)$ \\
\hline$c$ & 2 & $(1,3)(1,1)(0,-1)$ & 6 & -6 & - & - & - & - & $0(3)$ & $0(1)$ & -1 & 3 \\
\hline \hline$d$ & $3(\kappa-1)$ & $(1,0)(0,-2)(0,1)$ & 0 & 0 & - & - & - & - & - & $0(2)$ & $0(1)$ & $0(2)$ \\
\hline$e$ & $3(\kappa-1)$ & $(0,-1)(2,0)(0,1)$ & 0 & 0 & - & - & - & - & - & - & $0(2)$ & $0(1)$ \\
\hline$f$ & $3(\kappa-1)$ & $(0,-1)(0,2)(1,0)$ & 0 & 0 & - & - & - & - & - & - & - & $0(2)$ \\
\hline$O 3$ & 5 & $(1,0)(2,0)(1,0)$ & - & - & \multicolumn{5}{|c|}{$2 \chi_{3}=\chi_{2}=6 \chi_{1}$} \\
\hline
\end{tabular}

TABLE VIII: D-brane configurations and intersection numbers for a series of the models on Type IIB $\mathbf{T}^{\mathbf{6}}$ orientifold. The complete gauge symmetry is $U(4)_{C} \times U(2)_{L} \times U(2)_{R} \times U S p(10) \times U S p(6(\kappa-$ $1))^{3}$, and the SM fermions and Higgs fields arise from the intersections on the first two-torus. By the way, $l_{a}^{i}$ is equal to $m_{a}^{i}$ and $2 m_{a}^{i}$ for the rectangular and tilted two-torus, respectively.

Next, we consider the gauge coupling unification and moduli stabilization. The real parts of the dilaton and Kähler moduli in our model are [34]

$$
\begin{aligned}
& \operatorname{Re} s=\frac{\sqrt{6} e^{-\phi_{4}}}{4 \pi}, \operatorname{Re}_{1}=\frac{\sqrt{6} e^{-\phi_{4}}}{2 \pi}, \\
& \operatorname{Re}_{2}=\frac{\sqrt{6} e^{-\phi_{4}}}{12 \pi}, \operatorname{Ret}_{3}=\frac{\sqrt{6} e^{-\phi_{4}}}{6 \pi},
\end{aligned}
$$

where $\phi_{4}$ is the four-dimensional dilaton. From Eq. (68), we obtain that the SM gauge couplings are unified at the string scale as follows

$$
g_{S U(3)_{C}}^{-2}=g_{S U(2)_{L}}^{-2}=\frac{3}{5} g_{U(1)_{Y}}^{-2}=\frac{\sqrt{6} e^{-\phi_{4}}}{2 \pi} .
$$

Using the unified gauge coupling $g^{2} \simeq 0.513$ in supersymmetric SMs at the GUT scale, we get

$$
\phi_{4} \simeq-1.61
$$

For moduli stabilization, we first obtain $t$ from Eqs. (766) and (83)

$$
\operatorname{Re} t=\frac{3 \sqrt{6} e^{-\phi_{4}}}{4 \pi}, \operatorname{Im} t= \pm \sqrt{\frac{3 \beta h_{0}}{f g}-\frac{27 e^{-2 \phi_{4}}}{8 \pi^{2}}} .
$$

Thus, we have

$$
\begin{aligned}
& \operatorname{Im} s=-\frac{1}{3} \operatorname{Im} t+\frac{\beta}{g}, \\
& \operatorname{Re} u=-\frac{\sqrt{6} f e^{-\phi_{4}}}{4 \pi \beta}, \quad \operatorname{Im} u=\frac{f}{3 \beta} \operatorname{Im} t-\frac{f}{g} .
\end{aligned}
$$




\begin{tabular}{|c||c||c|c|c||c|}
\hline & Quantum Number & $Q_{4}$ & $Q_{2 L}$ & $Q_{2 R}$ & Field \\
\hline \hline$a b$ & $3 \times(4, \overline{2}, 1,1)$ & 1 & -1 & 0 & $F_{L}\left(Q_{L}, L_{L}\right)$ \\
$a c$ & $3 \times(\overline{4}, 1,2,1)$ & -1 & 0 & 1 & $F_{R}\left(Q_{R}, L_{R}\right)$ \\
$c_{S}$ & $6 \times(1,1, \overline{3}, 1,1)$ & 0 & 0 & -2 & $T_{R}^{i}$ \\
$c_{A}$ & $6 \times(1,1,1,1,1)$ & 0 & 0 & 2 & $S_{R}^{i}$ \\
$c O 3$ & $3 \times(1,1,2,10)$ & 0 & 0 & 1 & $X^{i}$ \\
\hline \hline$a c^{\prime}$ & $3 \times(4,1,2,1)$ & 1 & 0 & 1 & \\
& $3 \times(\overline{4}, 1, \overline{2}, 1)$ & -1 & 0 & -1 & \\
\hline \multirow{2}{*}{$b c$} & $6 \times(1,2, \overline{2}, 1)$ & 0 & 1 & -1 & $\Phi_{i}\left(H_{u}^{i}, H_{d}^{i}\right)$ \\
& $6 \times(1, \overline{2}, 2,1)$ & 0 & -1 & 1 & $\bar{\Phi}_{i}$ \\
\hline$b c^{\prime}$ & $1 \times(1,2,2,1)$ & 0 & 1 & 1 & $\Phi^{\prime}\left(H_{u}^{\prime}, H_{d}^{\prime}\right)$ \\
& $1 \times(1, \overline{2}, \overline{2}, 1)$ & 0 & -1 & -1 & $\bar{\Phi}^{\prime}$ \\
\hline$b b^{\prime}$ & $6 \times(1,1,1,1)$ & 0 & 2 & 0 & $S_{L}^{i}$ \\
& $6 \times(1, \overline{1}, 1,1)$ & 0 & -2 & 0 & $\bar{S}_{L}^{i}$ \\
\hline
\end{tabular}

TABLE IX: The chiral and vector-like superfields, and their quantum numbers under the gauge symmetry $S U(4)_{C} \times S U(2)_{L} \times S U(2)_{R} \times U S p(10)$. This is the exact spectrum for $\kappa=1$.

In this paper, we shall choose the unified gauge coupling at the string scale as the unified gauge coupling in the supersymmetric SM at the GUT scale, in other words, we choose $\phi_{4}=-1.61$. And then we obtain

$$
\operatorname{Re} s=0.975, \operatorname{Ret}_{1}=1.95, \operatorname{Re}_{2}=0.325, \quad \operatorname{Ret}_{3}=0.650 \text {. }
$$

In the following discussions, we always have the above values for $\operatorname{Re} s, \operatorname{Re}_{1}, \operatorname{Ret}_{2}$, and $\operatorname{Re} t_{3}$. So, we will not present them again. Also, we will assume that $k_{0}, k_{1}, k_{2}$ and $k_{3}$ are positive integers, and

$$
k_{0} k_{1}=3, \quad \eta= \pm 1, \quad \eta^{\prime}= \pm 1
$$

\section{A. Models with $\kappa=1$}

First, we consider the models with $\kappa=1$. Although this kind of models is the same as that in Ref. [34], we consider the generic solution to the flux consistent equations, and find a huge number of flux models. The general solution to the flux consistent equations is

$$
\begin{aligned}
& e=2 k_{0} \eta, \quad \bar{\beta}=-2 k_{1} \eta, \quad f=-2 k_{2} \eta^{\prime} \\
& \beta=2 k_{3} \eta^{\prime}, \quad g=\frac{2 k_{1} k_{2} \eta}{k_{3}}, \quad h_{0}=-\frac{6 k_{0} k_{2} \eta}{k_{3}},
\end{aligned}
$$




\begin{tabular}{|c||c||c|c|c|c|}
\hline Representation & Multiplicity & $U(1)_{a}$ & $U(1)_{b}$ & $U(1)_{c}$ & Field \\
\hline \hline$\left(\overline{4}_{a},(6(\kappa-1))_{e}\right)$ & 1 & -1 & 0 & 0 & $X_{a e}$ \\
$\left(4_{a},(6(\kappa-1))_{f}\right)$ & 1 & 1 & 0 & 0 & $X_{a f}$ \\
$\left.\left(\overline{2}_{b},(6(\kappa-1))_{d}\right)\right)$ & 3 & 0 & -1 & 0 & $X_{b d}^{i}$ \\
$\left(2_{b},(6(\kappa-1))_{e}\right)$ & 1 & 0 & 1 & 0 & $X_{b e}$ \\
$\left(\overline{2}_{c},(6(\kappa-1))_{f}\right)$ & 1 & 0 & 0 & -1 & $X_{c f}$ \\
\hline
\end{tabular}

TABLE X: The chiral and vector-like superfields, and their quantum numbers under the gauge symmetry $S U(4)_{C} \times S U(2)_{L} \times S U(2)_{R}$ and $U S p(6(\kappa-1))^{3}$. This is the additional spectrum due to $\kappa>1$.

where $k_{1} k_{2}$ and $3 k_{0} k_{2}$ are multiples of $k_{3}$.

There are three kinds of possible solutions that can satisfy $e \bar{\beta}=-12$, so, let us discuss them one by one in the following:

(1) For $k_{0}, k_{1}, k_{2}$ and $k_{3}$, we have

$$
k_{0}=3, \quad k_{1}=1, \quad k_{2}=n N, \quad k_{3}=N .
$$

So we obtain

$$
\begin{aligned}
& e=6 \eta, \quad \bar{\beta}=-2 \eta, \quad f=-2 n N \eta^{\prime}, \\
& \beta=2 N \eta^{\prime}, \quad g=2 n \eta, \quad h_{0}=-18 n \eta
\end{aligned}
$$

Choosing $\phi_{4}=-1.61$, we obtain

$$
\begin{aligned}
& \operatorname{Re} u=0.975 n, \quad \operatorname{Im} t \equiv \sum_{i=1}^{3} \operatorname{Im} t_{i}= \pm \sqrt{\frac{27}{n}-8.56} \\
& \operatorname{Im} s=-\frac{1}{3} \operatorname{Im} t+\frac{N \eta^{\prime}}{n \eta}, \quad \operatorname{Im} u=-\frac{n}{3} \operatorname{Im} t+\frac{N \eta^{\prime}}{\eta} .
\end{aligned}
$$

Thus, in order to have $(\operatorname{Im} t)^{2}>0$, we obtain $n<4$. And in the very large $N$ limit, we obtain that only $\operatorname{Im} s$ and $\operatorname{Im} u$ will become very large as follows

$$
\operatorname{Im} s \sim N, \quad \operatorname{Im} u \sim N
$$

(2) For $k_{0}, k_{1}, k_{2}$ and $k_{3}$, we have

$$
k_{0}=1, \quad k_{1}=3, \quad k_{2}=n N, \quad k_{3}=N .
$$


So we obtain

$$
\begin{aligned}
& e=2 \eta, \quad \bar{\beta}=-6 \eta, \quad f=-2 n N \eta^{\prime} \\
& \beta=2 N \eta^{\prime}, \quad g=6 n \eta, \quad h_{0}=-6 n \eta
\end{aligned}
$$

With $\phi_{4}=-1.61$, we find $(\operatorname{Im} t)^{2}<0$. So, this solution is not a correct solution.

(3) For $k_{0}, k_{1}, k_{2}$ and $k_{3}$, we have

$$
k_{0}=1, \quad k_{1}=3, \quad k_{2}=n N, \quad k_{3}=3 N .
$$

So we obtain

$$
\begin{aligned}
& e=2 \eta, \quad \bar{\beta}=-6 \eta, \quad f=-2 n N \eta^{\prime} \\
& \beta=6 N \eta^{\prime}, \quad g=2 n \eta, \quad h_{0}=-2 n \eta
\end{aligned}
$$

With $\phi_{4}=-1.61$, we obtain $n=1$ from $(\operatorname{Im} t)^{2}>0$. And we have

$$
\begin{aligned}
& \operatorname{Re} u=0.325, \quad \operatorname{Im} t= \pm 0.664, \\
& \operatorname{Im} s=\mp 0.221+\frac{3 N \eta^{\prime}}{\eta}, \quad \operatorname{Im} u=\mp 0.0738+\frac{N \eta^{\prime}}{\eta} .
\end{aligned}
$$

In the very large $N$ limit, we obtain that only $\operatorname{Im} s$ and $\operatorname{Im} u$ will become very large as follows

$$
\operatorname{Im} s \sim N, \quad \operatorname{Im} u \sim N
$$

\section{B. Infinity Flux Vacua for $\kappa>1$}

We consider the models with $\kappa>1$. Comparing to the above models, we have additional gauge symmetry $U S p(6(\kappa-1))^{3}$. There are four kinds of solutions to the flux consistent equations, and we will study them in the following:

(1) The first kind of solutions to the flux consistent equations is

$$
\begin{aligned}
& e=2 k_{0} \kappa \eta, \quad \bar{\beta}=-2 k_{1} \eta, \quad f=-2 k_{2} \eta^{\prime}, \\
& \beta=2 k_{3} \eta^{\prime}, \quad g=\frac{2 k_{1} k_{2} \eta}{k_{3}}, \quad h_{0}=-\frac{6 k_{0} k_{2} \kappa \eta}{k_{3}},
\end{aligned}
$$

where $k_{1} k_{2}$ and $3 k_{0} k_{2} \kappa$ are the multiples of $k_{3}$. In order to have positive $(\operatorname{Im} t)^{2}$, we obtain

$$
\frac{9 k_{0} k_{3} \kappa}{k_{1} k_{2}} \geq 8.56
$$

Thus, there exists a huge number of solutions to the flux consistent equations. 
Let us consider a special case with finite $k_{i}$ while very large $\kappa$, i.e., only $\kappa$ can be very large. In the very large $\kappa$ limit, we obtain that only $\operatorname{Im} s, \operatorname{Im} t$, and $\operatorname{Im} u$ will become very large as follows

$$
\operatorname{Im} s \sim \sqrt{\kappa}, \quad \operatorname{Im} t \sim \sqrt{\kappa}, \quad \operatorname{Im} u \sim \sqrt{\kappa} .
$$

In this case, let us present a concrete example. We choose

$$
\begin{array}{ll}
e=6 \kappa \eta, & \bar{\beta}=-2 \eta, \quad f=-2 \eta^{\prime}, \\
\beta=2 \eta^{\prime}, & g=2 \eta, \quad h_{0}=-18 \kappa \eta .
\end{array}
$$

With $\phi_{4}=-1.61$, we have

$$
\begin{aligned}
& \operatorname{Re} u=0.975, \quad \operatorname{Im} t= \pm \sqrt{27 \kappa-8.56} \\
& \operatorname{Im} s=\operatorname{Im} u=-\frac{1}{3} \operatorname{Im} t+\frac{\eta^{\prime}}{\eta} .
\end{aligned}
$$

(2) The second kind of solutions to the flux consistent equations is

$$
\begin{aligned}
& e=2 k_{0} \eta, \quad \bar{\beta}=-2 k_{1} \kappa \eta, \quad f=-2 k_{2} \eta^{\prime}, \\
& \beta=2 k_{3} \eta^{\prime}, \quad g=\frac{2 k_{1} k_{2} \kappa \eta}{k_{3}}, \quad h_{0}=-\frac{6 k_{0} k_{2} \eta}{k_{3}},
\end{aligned}
$$

where $k_{1} k_{2} \kappa$ and $3 k_{0} k_{2}$ are the multiples of $k_{3}$. In order to have positive $(\operatorname{Im} t)^{2}$, we obtain

$$
\frac{9 k_{0} k_{3}}{k_{1} k_{2} \kappa} \geq 8.56 \text {. }
$$

And then, we obtain

$$
\begin{gathered}
k_{3}=k_{1} k_{2} \kappa, \quad \text { for } k_{0}=1, \\
k_{3}=k_{1} k_{2} \kappa / n, \quad \text { for } \quad k_{0}=3,
\end{gathered}
$$

where $n=1,2,3$. Thus, there also exists a huge number of solutions to the flux consistent equations.

However, for very large $\kappa$ and finite $k_{i}$, we obtain that $(\operatorname{Im} t)^{2}$ is negative, and then we do not have such kind of solutions.

(3) The third kind of solutions to the flux consistent equations is

$$
\begin{aligned}
& e=2 k_{0} N \eta, \quad \bar{\beta}=-2 k_{1} N \eta, \quad f=-2 k_{2} N \eta^{\prime}, \\
& \beta=2 k_{3} N \eta^{\prime}, \quad g=\frac{2 k_{1} k_{2} N \eta}{k_{3}}, \quad h_{0}=-\frac{6 k_{0} k_{2} N \eta}{k_{3}},
\end{aligned}
$$


where $N$ is a positive integer, and $k_{1} k_{2} N$ and $3 k_{0} k_{2} N$ are the multiples of $k_{3}$. Also, we have

$$
\kappa=N^{2} .
$$

In order to have positive $(\operatorname{Im} t)^{2}$, we obtain

$$
\frac{9 k_{0} k_{3}}{k_{1} k_{2}} \geq 8.56 .
$$

Thus, there still exists a huge number of solutions to the flux consistent equations. In fact, we just rescale all the fluxes by $N$. Note that the flux ratios are independent on $N$, we obtain that $\operatorname{Re} u, \operatorname{Im} s, \operatorname{Im} t$ and $\operatorname{Im} u$ are constants since they only depend on the flux ratios. In this case, all the phenomenological discussions are similar to those in Ref. [34] except that we have additional $U S p(6 \kappa-6)^{3}$ gauge symmetry and the corresponding extra chiral exotic particles in Table $\mathrm{X}$.

Let us give a concrete example for finite $k_{i}$ and very large $N$. We choose

$$
\begin{aligned}
& e=6 N \eta, \quad \bar{\beta}=-2 N \eta, \quad f=-2 N \eta^{\prime}, \\
& \beta=2 N \eta^{\prime}, \quad g=2 N \eta, \quad h_{0}=-18 N \eta .
\end{aligned}
$$

With $\phi_{4}=-1.61$, we have

$$
\operatorname{Re} u=0.975, \quad \operatorname{Im} t= \pm 4.30, \quad \operatorname{Im} s=\operatorname{Im} u=\mp 1.43+1 .
$$

This is similar to the example in Ref. [34.

(4) The fourth kind of solutions to the flux consistent equations is

$$
\begin{aligned}
& e=2 k_{0} N \eta, \quad \bar{\beta}=-2 k_{1} N \eta, \quad f=-2 k_{2} \eta^{\prime}, \\
& \beta=2 k_{3} N \eta^{\prime}, \quad g=\frac{2 k_{1} k_{2} \eta}{k_{3}}, \quad h_{0}=-\frac{6 k_{0} k_{2} \eta}{k_{3}},
\end{aligned}
$$

where $k_{1} k_{2}$ and $3 k_{0} k_{2}$ are the multiples of $k_{3}$. Also, we have $\kappa=N^{2}$. In order to have positive $(\operatorname{Im} t)^{2}$, we obtain

$$
\frac{9 k_{0} k_{3} N}{k_{1} k_{2}} \geq 8.56 .
$$

So, we also have a huge number of solutions to the flux consistent equations.

If $N$ is very large while $k_{i}$ is finite, we obtain that $\operatorname{Re} u, \operatorname{Im} s, \operatorname{Im} t$, and $\operatorname{Im} u$ will depend on $N$ as follows

$$
\operatorname{Re} u \sim \frac{1}{N} \sim \frac{1}{\sqrt{\kappa}}, \quad \operatorname{Im} s \sim N \sim \sqrt{\kappa}, \quad \operatorname{Im} t \sim \sqrt{N} \sim \kappa^{1 / 4}, \quad \operatorname{Im} u \sim \frac{1}{\sqrt{N}} \sim \frac{1}{\kappa^{1 / 4}} .
$$


Thus, in the very large $N$ limit, $\operatorname{Re} u$ and $\operatorname{Im} u$ will be very small while $\operatorname{Im} s$ and $\operatorname{Im} t$ will be very large. Let us present a concrete example for this case. We choose

$$
\begin{aligned}
& e=6 N \eta, \quad \bar{\beta}=-2 N \eta, \quad f=-2 \eta^{\prime}, \\
& \beta=2 N \eta^{\prime}, \quad g=2 \eta, \quad h_{0}=-18 \eta .
\end{aligned}
$$

With $\phi_{4}=-1.61$, we have

$$
\begin{aligned}
& \operatorname{Re} u=\frac{0.975}{N}, \quad \operatorname{Im} t= \pm \sqrt{27 N-8.56} \\
& \operatorname{Im} s=-\frac{1}{3} \operatorname{Im} t+\frac{N \eta^{\prime}}{\eta}, \quad \operatorname{Im} u=-\frac{1}{3 N} \operatorname{Im} t+\frac{\eta^{\prime}}{\eta} .
\end{aligned}
$$

\section{DISCUSSION AND CONCLUSIONS}

In this paper, we studied the detail flux parameter spaces for semi-realistic supersymmetric Pati-Salam models in the AdS vacua on Type IIA orientifold and realistic supersymmetric Pati-Salam models in the Minkowski vacua on Type IIB orientifold with general flux compactifications. We have shown that there indeed exists a huge number of semi-realistic Type IIA and realistic Type IIB flux models. So, we do have the string landscape for the flux models. However, these semi-realistic Tyep IIA and realistic Type IIB flux models can not be populated in the string landscape.

For the supersymmetric intersecting D6-brane model building in the AdS vacua on Type IIA orientifold with flux compactifications, if we keep $\left(e_{0} a-e h_{0}\right)$ as a constant while allowing $e$ and/or $e_{0}$ to be very large, the very large fluxes do not affect the main properties of the models, and only ( $\left.3 a \operatorname{Im} s+\sum_{i=1}^{3} b_{i} \operatorname{Im} u_{i}\right)$ will be proportional to $e$ and then very large. Also, if $\left(e_{0} a-e h_{0}\right)$ is very large, we showed that the theory is very weakly coupled, and the magnitude of the cosmological constant becomes very small. And if only $e_{0}$ is very large, $\left(3 a \operatorname{Im} s+\sum_{i=1}^{3} b_{i} \operatorname{Im} u_{i}\right)$ will be a constant as well since it does not depend on $e_{0}$. Moreover, we considered two kinds of semi-realistic Pati-Salam models with very large fluxes $a, h_{0}$, $m$, and/or $q$. In the first kind of models, we keep $\left(h_{0} m+3 q a\right)$ as a negative constant. So we do not change the RR tadpole cancellation conditions, the gauge symmetries, and the particle spectra of the models due to the very large fluxes. In the very large flux limit, the theory becomes very weakly coupled, and the magnitude of the cosmological constant will become very small as well. In the second kind of Pati-Salam models, we took not only a and $h_{0}$ to be very large, but also $\left(h_{0} m+3 q a\right)$ to be negative and very large. In particular, we took the complex structure moduli to be very large so that only one of the RR tadpole cancellation conditions is very large and proportional to $a$ or $h_{0}$. We showed that the gauge symmetry can be broken down to the SM gauge symmetry, and the exotic particles might 
be decoupled. The gauge couplings for $S U(2)_{L}$ and $S U(2)_{R}$ are half of these for $S U(3)_{C}$ and $U(1)_{B-L}$. In the very large flux limit, the gauge coulings approach to the fixed constants. However, the magnitude of the cosmological constant will be very large, which can become very small again if we introduce very large fluxes $e$ and/or $e_{0}$.

For the supersymmetric D-brane model building in the Minkowski vacua on Type IIB orientifold with general flux compactifications, we constructed a series of realistic Pati-Salam models with gauge group $U(4)_{C} \times U(2)_{L} \times U(2)_{R} \times U S p(10) \times U S p(6(\kappa-1))^{3}$. Interestingly, in the very large flux limit, we can choose the unified gauge coupling at the string scale as the unified gauge coupling in the supersymmetric SMs at the GUT scale because the real parts of the dilaton and Kähler moduli can be independent of the very large fluxes. For the first kind of models with $\kappa=1$, the very large fluxes do not contribute to the RR tadpole cancellation conditions, and we found two kinds of solutions to the flux consistent equations. In the very large flux limit, the real part of the complex structure moduli and the sum of the imaginary parts of the Kähler moduli are constants and independent of the very large fluxes. Only the imaginary parts of the dilaton and complex structure moduli become very large and proportional to the very large fluxes. For the second kind of models with $\kappa>1$, we obtained four kinds of solutions to the flux consistent equations, and showed that there indeed exists a huge number of the realistic flux models. In the very large $\kappa$ limit, we consider the asymptotic behaviour for $\operatorname{Re} u, \operatorname{Im} s, \operatorname{Im} t$, and $\operatorname{Im} u$, and present some concrete examples.

\section{Acknowledgments}

This research was supported in part by the Mitchell-Heep Chair in High Energy Physics (CMC), by the Cambridge-Mitchell Collaboration in Theoretical Cosmology (TL), and by the DOE grant DE-FG03-95-Er-40917 (DVN).

[1] J. Polchinski and E. Witten, Nucl. Phys. B 460, 525 (1996).

[2] M. Berkooz, M. R. Douglas and R. G. Leigh, Nucl. Phys. B 480, 265 (1996).

[3] C. Bachas, arXiv:hep-th/9503030.

[4] R. Blumenhagen, L. Goerlich, B. Kors and D. Lust, JHEP 0010, 006 (2000).

[5] C. Angelantonj, I. Antoniadis, E. Dudas and A. Sagnotti, Phys. Lett. B 489, 223 (2000).

[6] L. E. Ibanez, F. Marchesano and R. Rabadan, JHEP 0111, 002 (2001). 
[7] R. Blumenhagen, M. Cvetic, P. Langacker and G. Shiu, Ann. Rev. Nucl. Part. Sci. 55, 71 (2005), and references therein.

[8] M. Cvetič, G. Shiu and A. M. Uranga, Phys. Rev. Lett. 87, 201801 (2001).

[9] M. Cvetič, G. Shiu and A. M. Uranga, Nucl. Phys. B 615, 3 (2001).

[10] M. Cvetič and I. Papadimitriou, Phys. Rev. D 67, 126006 (2003).

[11] M. Cvetič, I. Papadimitriou and G. Shiu, Nucl. Phys. B 659, 193 (2003) [Erratum-ibid. B 696, 298 (2004)].

[12] M. Cvetič, T. Li and T. Liu, Nucl. Phys. B 698, 163 (2004).

[13] M. Cvetič, P. Langacker, T. Li and T. Liu, Nucl. Phys. B 709, 241 (2005).

[14] C.-M. Chen, G. V. Kraniotis, V. E. Mayes, D. V. Nanopoulos and J. W. Walker, Phys. Lett. B 611, 156 (2005); Phys. Lett. B 625, 96 (2005).

[15] C. M. Chen, T. Li and D. V. Nanopoulos, Nucl. Phys. B 732, 224 (2006).

[16] R. Blumenhagen, L. Görlich and T. Ott, JHEP 0301, 021 (2003); G. Honecker, Nucl. Phys. B666, 175 (2003); G. Honecker and T. Ott, Phys. Rev. D 70, 126010 (2004) [Erratum-ibid. D 71, $069902(2005)]$.

[17] C. M. Chen, T. Li, V. E. Mayes and D. V. Nanopoulos, arXiv:hep-th/0703280.

[18] C. M. Chen, T. Li, V. E. Mayes and D. V. Nanopoulos, arXiv:0711.0396 [hep-ph].

[19] M. Cvetič, P. Langacker and J. Wang, Phys. Rev. D 68, 046002 (2003).

[20] S. Gukov, C. Vafa and E. Witten, Nucl. Phys. B 584, 69 (2000) [Erratum-ibid. B 608, 477 (2001)].

[21] S. B. Giddings, S. Kachru and J. Polchinski, Phys. Rev. D 66, 106006 (2002).

[22] S. Kachru, M. B. Schulz, P. K. Tripathy and S. P. Trivedi, JHEP 0303, 061 (2003).

[23] S. Kachru, R. Kallosh, A. Linde and S. P. Trivedi, Phys. Rev. D 68, 046005 (2003).

[24] J. F. G. Cascales and A. M. Uranga, JHEP 0305, 011 (2003).

[25] R. Blumenhagen, D. Lüst and T. R. Taylor, Nucl. Phys. B 663, 319 (2003).

[26] F. Marchesano and G. Shiu, Phys. Rev. D 71, 011701 (2005); JHEP 0411, 041 (2004).

[27] M. Cvetič and T. Liu, Phys. Lett. B 610, 122 (2005).

[28] M. Cvetič, T. Li and T. Liu, Phys. Rev. D 71, 106008 (2005).

[29] J. Kumar and J. D. Wells, JHEP 0509, 067 (2005).

[30] C. M. Chen, V. E. Mayes and D. V. Nanopoulos, Phys. Lett. B 633, 618 (2006).

[31] J. Shelton, W. Taylor and B. Wecht, JHEP 0510, 085 (2005).

[32] G. Aldazabal, P. G. Camara, A. Font and L. E. Ibanez, JHEP 0605, 070 (2006).

[33] G. Villadoro and F. Zwirner, JHEP 0603, 087 (2006). 
[34] C. M. Chen, T. Li, Y. Liu and D. V. Nanopoulos, arXiv:0711.2679 [hep-th].

[35] T. W. Grimm and J. Louis, Nucl. Phys. B 718, 153 (2005).

[36] G. Villadoro and F. Zwirner, JHEP 0506, 047 (2005).

[37] P. G. Camara, A. Font and L. E. Ibanez, JHEP 0509, 013 (2005).

[38] C. M. Chen, T. Li and D. V. Nanopoulos, Nucl. Phys. B 740, 79 (2006).

[39] C. M. Chen, T. Li and D. V. Nanopoulos, Nucl. Phys. B 751, 260 (2006).

[40] L. Susskind, arXiv:hep-th/0302219.

[41] F. Denef and M. R. Douglas, JHEP 0405, 072 (2004).

[42] S. Weinberg, Phys. Rev. Lett. 59, 2607 (1987).

[43] F. Gmeiner, R. Blumenhagen, G. Honecker, D. Lust and T. Weigand, JHEP 0601, 004 (2006).

[44] R. Blumenhagen, B. Körs and D. Lüst, JHEP 0102 (2001) 030.

[45] E. Witten, JHEP 9812, 019 (1998).

[46] F. G. Marchesano Buznego, hep-th/0307252.

[47] D. S. Freed and E. Witten, arXiv:hep-th/9907189.

[48] D. Cremades, L. E. Ibanez and F. Marchesano, JHEP 0207, 009 (2002).

[49] D. Lust, P. Mayr, R. Richter and S. Stieberger, Nucl. Phys. B 696, 205 (2004).

[50] T. Li, Phys. Lett. B 653, 338 (2007). 